\title{
Deformation Behaviour of Stomatocyte, Discocyte and Echinocyte RBC Morphologies during Optical Tweezers Stretching
}

\author{
NM Geekiyanage, E Sauret, SC Saha, RL Flower, and YT Gu
}

\begin{abstract}
The red blood cell (RBC) deformability is a critical aspect, and assessing the cell deformation characteristics is essential for better diagnostics of healthy and deteriorating RBCs. There is a need to explore the connection between the cell deformation characteristics, cell morphology, disease states, storage lesion and cell shape-transformation conditions for better diagnostics and treatments. A numerical approach inspired from the previous research for RBC morphology predictions and for analysis of RBC deformations, is proposed for the first time, to investigate the deformation characteristics of different RBC morphologies. The present study investigates the deformability characteristics of stomatocyte, discocyte and echinocyte morphologies during optical tweezers stretching, and provides the opportunity to study the combined contribution of cytoskeletal spectrin network and the lipid-bilayer during RBC deformation. The proposed numerical approach predicts agreeable deformation characteristics of the healthy discocyte with the analogous experimental observations, and is extended to further investigate the deformation characteristics of stomatocyte and echinocyte morphologies. In particular, the computer simulations are performed to investigate the influence of direct stretching forces on different equilibrium cell morphologies on cell spectrin link extensions and cell elongation index, along with a parametric analysis on membrane shear modulus, spectrin link extensibility, bending modulus and RBC membrane-bead contact diameter. The results confirm the lowered deformability of stomatocyte and echinocyte morphologies with respect to a healthy discocyte, and suggest the preservation of relevant morphological characteristics, changes in spectrin link densities and the primary contribution of cytoskeletal spectrin network on deformation behaviour of stomatocyte, discocyte and echinocyte morphologies during optical tweezers stretching deformation. The numerical approach presented here forms the foundation for investigations into deformation characteristics and recoverability of RBCs undergoing storage lesion.
\end{abstract}

Keywords: Red blood cell, Optical tweezers, Numerical modelling, Coarse-grained, Elongation index Abbreviation:

ATP: adenosine-tri-phosphate; BCM: bilayer-couple model; CG: coarse-grained; EI: elongation index; RBC: red blood cells; SDE: stomatocyte-discocyte-echinocyte 


\section{Introduction}

Red blood cells (RBCs) though remarkably simple in structure [1], perform the vital physiological function of transferring oxygen and carbon dioxide between lung and body tissues. RBCs require high deformability to sustain its passage through narrow capillaries of the microvasculature, which is primarily influenced by mechanical and geometrical factors of the cell such as cell surface area and volume, elasticity and viscosity of the cell membrane, and volume and viscosity of the cytosol [2-7]. Therefore, any change in RBC morphology would indicate altered deformability, and certain pathophysiological conditions such as hereditary haemolytic disorders (i.e., spherocytosis, elliptocytosis and ovalocytosis) [1, 2], sickle cell disease, malaria [8-15], shape-transforming agents (i.e., stomatocytogenic and echinocytogenic agents) [16-22] and in-vitro RBC storage [23-26] can alter its healthy biconcave discocyte morphology.

These RBC disease conditions can affect the inter-protein and protein-to-lipid linkages of the cell membrane [27], and can alter the mechanical and structural integrity of the cell membrane. The cell deformability is adversely affected during storage as well. The viscosity and the viscoelastic time constant of the cell membrane increase with in-vitro storage duration, and the cell becomes denser and smaller as well [25]. The Young's modulus of the cells significantly increases with storage duration as well [28], indicating decreased cell deformability. In addition, gradual depletion of adenosine tri phosphate (ATP) occurs during storage, and the RBC shape is also influenced by the cell metabolic activities that regulate the intracellular ATP concentration [4, 29-31]. The ATP-depleted RBCs indicate increased cell stiffness [4, 23, 32]. The stomatocytogenic and echinocytogenic shape-transforming conditions can transform the usual biconcave discocyte shape into stomatocytes and echinocytes, respectively at constant cell surface area and volume [17-21]. These shape changes are reversible to the stage where sphero-stomatocyte and sphero-echinocyte are formed [33]. However, altered membrane shear modulus and bending modulus have been observed with the stomatocyte-discocyte-echinocyte (SDE) shape-transformations of a RBC. Therefore, it has been suggested [15] that the cell shapetransformations are accompanied by modifications of either the spectrin elasticity, the connectivity of the cytoskeletal spectrin network or its attachment to the lipid-bilayer. It has also been suggested [34] that the RBC cytoskeletal spectrin network is continuously rearranged due to its metabolic activity or large strains such that strain hardening and softening of the membrane may occur. The deformability of the cell can therefore be used as a criterion to examine the health of a RBC subjected to morphological, structural and functional changes, and deformability investigations provide valuable insights into the physiology, cell biology and biorheology under such pathophysiological conditions.

Different techniques have been used to investigate the RBC deformability in vitro, and descriptions of these techniques can be found in $[4,10,11,15,29,34-43]$. Optical tweezers is one such technique and provides a highly sensitive assessment of the cell deformability at the single cell level. With optical tweezers, it is possible to trap, manipulate and displace a living cell or a part of it without damage, either 
directly or using specific handles such as dielectric beads of silica $[2,8,25,44,45]$. The RBC membrane is primarily responsible for the cell morphology and its elastic response during optical tweezers stretching deformation as the intracellular fluid is purely viscous and has no elasticity [44]. Furthermore, Sheetz and Singer [46] proposed the widely known bilayer-couple hypothesis to explain the SDE transformation, and discussed the contribution of the two leaflets of the lipid bilayer towards RBC morphology transformations. Several research studies have investigated the SDE transformation experimentally and numerically $[3,18,19,22,33,47-59]$.

Even though, the cell deformability is linked with its morphology, the investigations to understand the contribution of cell morphology towards its deformation characteristics are limited. However, improved understanding on the link between the cell morphology and its deformation characteristics is essential for better diagnostics of healthy and deteriorating RBCs. Therefore, a framework is in need to explore the interrelation between cell morphology, deformation characteristics, disease states, storage lesion and cell shape-transformation conditions for improved diagnostics and treatments. Numerical modelling is a desirable approach to investigate the fundamental mechanics of cell deformability over the experimental investigations, which can be challenging and costly [43]. This paper proposes a numerical approach inspired from the previous research for RBC morphology predictions [54, 60, 61] and for analysis of RBC deformations [1,34] to investigate, for the first time, the deformation characteristics of different RBC morphologies. A three-dimensional coarse-grained (CG) $-\mathrm{RBC}$ membrane model based on the bilayer-couple model (BCM) is employed to predict the deformation characteristics of stomatocyte, discocyte and echinocyte morphologies of a RBC during optical tweezers stretching, and provides the opportunity to study the combined contribution of cytoskeletal spectrin network and the lipid-bilayer during cell deformation. The numerically observed deformation characteristics of the healthy discocyte is validated against the analogous experimental observations, and the numerical approach is then extended to investigate the deformation characteristics of stomatocyte and echinocyte morphologies. The evolution of cell shape, cell axial and transverse diameters, elongation index and extension of spectrin links of above RBC morphologies subjected to varied stretching forces, are investigated. In addition, a parametric analysis of RBC membrane shear modulus, bending modulus, spectrin link extensibility, and RBC membrane-bead contact diameter is performed to examine the corresponding influence on cell deformation characteristics. Following subsections briefly outline the coarse-grained (CG)-RBC membrane model used for the shape predictions, implementation of optical tweezers stretching on the RBC membrane and discuss the influence of morphology on RBC deformability characteristics. Furthermore, this numerical approach is currently being executed to investigate the deformability characteristics and recoverability of RBCs undergoing storage lesion. 


\section{Methods}

In the first step, the CG-RBC membrane model is employed to predict the stomatocyte, discocyte and echinocyte morphologies. The CG-RBC membrane model is briefly reviewed below, whereas detailed description and discussion on the model development and shape predictions are available in Geekiyanage et al. [61]. In the second step, the deformation behaviour of the above RBC morphologies is investigated during optical tweezers stretching conditions.

\subsection{CG-RBC Membrane Model}

The CG-RBC membrane model is composed of $N_{V}$ vertices to represent the cytoskeletal actin junctional complexes and form a two-dimensional triangulated surface of $N_{t}$ triangles. The $N_{S}$ adjacent vertexvertex connections represent the cytoskeletal spectrin links. The free-energy of the CG-RBC membrane model $(E)$ takes into account the elastic energy of the cytoskeletal spectrin network $\left(E_{\text {Stretching }}\right)$, bending resistance of the lipid-bilayer $\left(E_{\text {Bending }}\right)$, and constraints of fixed total surface area ( $\left.E_{\text {Surface-area }}\right)$ and enclosed volume $\left(E_{\text {Volume }}\right)$. A bilayer-couple model (BCM)-based membrane bending energy approach $[49,50,53,62,63]$ is implemented via bilayer-leaflet-area-difference constraint $\left(E_{\text {Area-difference }}\right)$ to achieve the stomatocyte, discocyte and echinocyte morphologies at corresponding reference bilayer-leaflet-area-difference $\left(\Delta A_{0}\right)$. In addition, the total-membranecurvature constraint $\left(E_{\text {Total-curvature }}\right)$ is implemented to achieve numerically consistent RBC morphologies at corresponding reference total-membrane-curvature $\left(C_{0}\right)$. Therefore, $E$ is given by,

$$
E=E_{\text {Stretching }}+E_{\text {Bending }}+E_{\text {Surface-area }}+E_{\text {Volume }}+E_{\text {Area-difference }}+E_{\text {Total-curvature }}
$$

$E_{\text {Stretching }}$ is estimated based on the coarse-graining approach by Fedosov et al. [34], and is given by,

$$
E_{\text {Stretching }}=\sum_{j \in 1 \ldots N_{S}}\left[\frac{k_{B} T l_{\max }}{4 p} \frac{3 x_{j}^{3}-2 x_{j}^{3}}{1-x_{j}}+\frac{k_{p}}{(m-1) l_{j}^{m-1}}\right]
$$

where, $k_{B}$ is the Boltzmann constant, $T$ is the absolute temperature, $l_{\max }$ is the maximum extension of $j^{\text {th }}$ link, $p$ is the persistence length and $k_{p}$ is the power function coefficient. $x_{j}$ is defined as $x_{j}=$ $l_{j} / l_{\max }$ for the $j^{t h}$ link having $l_{j}$ length, and $m$ is an exponent such that $m>1$. The experimentally estimated membrane shear modulus $\left(\mu_{0}\right)$ of a healthy RBC lies between $4-12 \mu \mathrm{Nm}^{-1}[34,63]$, and can be determined as below for the CG-RBC membrane model [34].

$$
\mu_{0}=\frac{\sqrt{3} k_{B} T}{4 p l_{\max } x_{0}}\left[\frac{x_{0}}{2\left(1-x_{0}\right)^{3}}-\frac{1}{4\left(1-x_{0}\right)^{2}}-\frac{1}{4}\right]+\frac{\sqrt{3} k_{p}(m+1)}{4 l_{0}^{m+1}}
$$

where, $l_{0}$ is the equilibrium length of $j^{\text {th }}$ link, and defined as $x_{0}=l_{0} / l_{\max } . E_{\text {Bending }}$ of the lipidbilayer for a zero spontaneous curvature, is estimated by, 


$$
E_{\text {Bending }}=2 \kappa \sum_{j \in 1 \ldots N_{S}} \frac{M_{j}^{2}}{\Delta A_{j}}
$$

where, $\kappa$ is the membrane bending modulus, $M_{j}$ is the membrane curvature at $j^{\text {th }}$ link, and $\Delta A_{j}$ is the membrane surface area associated with the $j^{\text {th }}$ link. $M_{j}$ and $\Delta A_{j}$ corresponding to the triangle-pair composed of $T 1$ and $T 2$ triangles that shares the $j^{\text {th }}$ link, are estimated as follows;

$$
\begin{aligned}
& M_{j}=\frac{1}{2} l_{j} \theta_{j} \\
& \Delta A_{j}=\frac{1}{3}\left(A_{T 1}+A_{T 2}\right)
\end{aligned}
$$

where, $\theta_{j}$ is the angle between outward normal vectors to the triangles $T 1$ and $T 2$, and $A_{T 1}$ and $A_{T 2}$ are the planer area associated with $T 1$ and $T 2$, respectively. $\theta_{j}$ is defined such that the concave arrangement of $T 1$ and $T 2$ corresponds to a positive $\theta_{j}$, whereas the convex arrangement corresponds to a negative $\theta_{j}$, and results in positive or negative $M_{j}$, respectively.

The energy components due to constraints: $E_{\text {Surface-area }}, E_{\text {Volume }}, E_{\text {Area-difference }}$ and $E_{\text {Total-curvature }}$, are estimated by [34, 61, 64],

$$
\begin{gathered}
E_{\text {Surface-area }}=\frac{1}{2} k_{A}\left(\frac{A-A_{0}}{A_{0}}\right)^{2} A_{0}+\sum_{k \in 1 \ldots N_{t}} \frac{1}{2} k_{a}\left(\frac{A_{k}-A_{k, 0}}{A_{k, 0}}\right)^{2} A_{k, 0} \\
E_{\text {Volume }}=\frac{1}{2} k_{V}\left(\frac{V-V_{0}}{V_{0}}\right)^{2} V_{0} \\
E_{\text {Area-difference }}=\frac{1}{2} \frac{\pi k_{A D}}{D_{0}^{2}}\left(\frac{\Delta A-\Delta A_{0}}{A}\right)^{2} A \\
E_{\text {Total-curvature }}=\frac{1}{2} \frac{\pi k_{C}}{D_{0}^{2}}\left(\frac{C-C_{0}}{A}\right)^{2} A
\end{gathered}
$$

where, $A_{0}$ is the reference membrane surface area, $A$ is the instantaneous membrane surface area, $A_{k, 0}$ is the reference area of $k^{t h}$ triangle, and $A_{k}$ is the instantaneous area of $k^{\text {th }}$ triangle. $V_{0}$ is the reference cell volume and $V$ is the instantaneous cell volume. $D_{0}$ is the monolayer thickness of the lipid-bilayer, $\Delta A$ is the instantaneous bilayer-leaflet-area-difference, and $C$ is the instantaneous total-membranecurvature. $k_{A}, k_{a}, k_{V}, k_{A D}$ and $k_{C}$ represent the total surface area, local surface area, volume, bilayerleaflet-area-difference and total-membrane-curvature constraint coefficients, respectively.

It is assumed that the membrane vertices move over the RBC membrane surface to achieve the minimum free energy state, which is the equilibrium RBC shape. The force $\left(F_{i}\right)$ acting on the $i^{\text {th }}$ vertex at point $r_{i}$ on the surface is derived from the principle of virtual work, such that;

$$
F_{i}=-\frac{\partial E}{\partial r_{i}}, \quad i \in 1 \ldots N_{V}
$$


The resulting motion of the $i^{\text {th }}$ vertex is then estimated from the Newton's second law of motion as follows;

$$
F_{i}+f_{i}^{e x t}=m_{i} \ddot{r}_{i}+c \dot{r}_{i}
$$

where, $f_{i}^{e x t}$ is the contribution from any external forces on $i^{\text {th }}$ vertex point, $m_{i}$ is the mass of $i^{\text {th }}$ vertex point, dot (.) is the time derivative and $c$ is the viscosity of the RBC membrane. The updated velocity $\left(\dot{r}_{i}\right)$ and the position $\left(r_{i}\right)$ of the $i^{t h}$ vertex at time $(t+\Delta t)$ from the time $(t)$ is given as;

$$
\begin{aligned}
& \dot{r}_{i}(t+\Delta t)=c \dot{r}_{i}(t)+\ddot{r}_{i}(t) \Delta t \\
& r_{i}(t+\Delta t)=r_{i}(t)+\dot{r}_{i}(t+\Delta t) \Delta t
\end{aligned}
$$

The iterations are continued until the RBC membrane reaches the equilibrium state, which is the minimum free energy state of the RBC membrane at given reference conditions. In the present computational implementation, the equilibrium cell state is acknowledged and the derivation is terminated when the change between each analogous energy component at two successive iterations, is less than $1 \times 10^{-7}$ in the order of energy component in consideration.

\subsection{Prediction of Stomatocyte, Discocyte and Echinocyte RBC Morphologies}

The CG-RBC membrane model predicted stomatocyte II, discocyte and echinocyte II morphologies, which each generally represent stomatocyte, discocyte and echinocyte, respectively, are achieved according to the method presented in Geekiyanage et al. [61], and the key model parameters are summarised in Table 1. The analogous equilibrium stomatocyte, discocyte and echinocyte shapes are presented in Figure 1.

Table 1 - The key parameters values used to model the stomatocyte, discocyte and echinocyte RBC morphologies.

\begin{tabular}{|c|l|c|c|}
\hline Parameter & \multicolumn{1}{|c|}{ Description } & Value & Source \\
\hline$A_{0}$ & Reference RBC membrane surface area & $140.0 \mu \mathrm{m}^{2}$ & $\begin{array}{c}{[29,61,} \\
65]\end{array}$ \\
\hline$C_{0, \text { Discocyte }}$ & Reference total-membrane-curvature for discocyte & $1.85 \times 10^{-3} A_{0} \mathrm{~m}^{2}$ & {$[61]$} \\
\hline$C_{0, \text { Echinocyte }}$ & Reference total-membrane-curvature for echinocyte & $5.50 \times 10^{-3} A_{0} \mathrm{~m}^{2}$ & {$[61]$} \\
\hline$C_{0, \text { Stomatocyte }}$ & Reference total-membrane-curvature for stomatocyte & $1.75 \times 10^{-3} A_{0} \mathrm{~m}^{2}$ & {$[61]$} \\
\hline$D_{0}$ & Monolayer thickness of the lipid-bilayer & $2.0 \mathrm{~nm}$ & {$[60,61]$} \\
\hline$N_{S}$ & Number of links in CG-RBC membrane model & 7,680 & {$[61]$} \\
\hline$N_{V}$ & Number of vertices in CG-RBC membrane model & 2,562 & {$[61]$} \\
\hline
\end{tabular}




\begin{tabular}{|c|c|c|c|}
\hline$N_{t}$ & Number of triangles in CG-RBC membrane model & 5,120 & [61] \\
\hline$V_{0}$ & Reference RBC volume & $93.48 \mu \mathrm{m}^{3}$ & $\begin{array}{l}{[29,61,} \\
65]\end{array}$ \\
\hline$k_{A}$ & $\begin{array}{l}\text { Constraint coefficient for total membrane surface } \\
\text { area }\end{array}$ & $1.0 \times 10^{-3} \mathrm{Nm}^{-1}$ & [61] \\
\hline$k_{A D}$ & $\begin{array}{l}\text { Constraint coefficient for bilayer-leaflet-area- } \\
\text { difference }\end{array}$ & $300.0 \kappa \mathrm{Nm}$ & {$[61]$} \\
\hline$k_{C}$ & Constraint coefficient for total-membrane-curvature & $100.0 \kappa \mathrm{Nm}$ & [61] \\
\hline$k_{V}$ & Constraint coefficient for cell volume & $100.0 \mathrm{Nm}^{-2}$ & [61] \\
\hline$k_{a}$ & $\begin{array}{l}\text { Constraint coefficient for local membrane surface } \\
\text { area }\end{array}$ & $5.0 \times 10^{-5} \mathrm{Nm}^{-1}$ & [61] \\
\hline$m_{i}$ & Mass of the $i^{\text {th }}$ CG-RBC membrane vertex & $1.0 \times 10^{-9} \mathrm{~kg}$ & [61] \\
\hline$x_{0}$ & Extensibility of spectrin links & 0.45 & {$[34,61]$} \\
\hline$\mu_{0}$ & Membrane shear modulus & $4.0 \mu \mathrm{Nm}^{-1}$ & $\begin{array}{c}{[34,61,} \\
63]\end{array}$ \\
\hline$\Delta A_{0, \text { Discocyte }}$ & Reference total-membrane-curvature for discocyte & $1.20 \times 10^{-3} A_{0} \mathrm{~m}^{2}$ & [61] \\
\hline$\Delta A_{0, \text { Echinocyte }}$ & Reference total-membrane-curvature for echinocyte & $1.90 \times 10^{-3} A_{0} \mathrm{~m}^{2}$ & [61] \\
\hline$\Delta A_{0, \text { Stomatocyte }}$ & Reference total-membrane-curvature for stomatocyte & $1.05 \times 10^{-3} A_{0} \mathrm{~m}^{2}$ & [61] \\
\hline$T$ & Absolute temperature & $296.15 \mathrm{~K}$ & [61] \\
\hline$c$ & Membrane viscosity & $1.0 \times 10^{-7} \mathrm{Nsm}^{-1}$ & [61] \\
\hline$m$ & Exponent of $E_{\text {Stretching }}$ & 2 & {$[34,61]$} \\
\hline$\kappa$ & Membrane bending modulus & $2.5 \times 10^{-19} \mathrm{Nm}$ & $\begin{array}{c}{[35,61,} \\
63]\end{array}$ \\
\hline
\end{tabular}

(a)

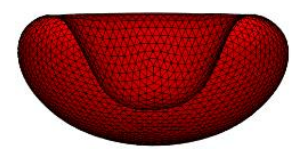

(b)

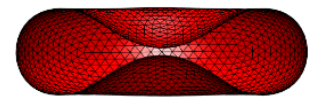

(c)

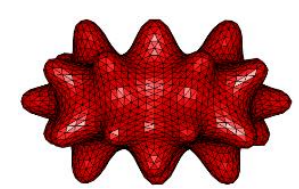

Figure 1 - The CG-RBC membrane model predicted (a) stomatocyte, (b) discocyte, and (c) echinocyte morphologies at model parameters given in Table 1. 


\subsection{Implementation of Optical Tweezers Stretching}

The optical tweezers stretching forces are implemented on CG-RBC membrane model predicted equilibrium stomatocyte, discocyte and echinocyte RBC morphologies. Assuming rigid body conditions, the centre of mass of the whole cell and its three-principal axes of inertia are determined for each RBC morphology. The total stretching force $\left(F^{\text {ext }}\right)$ is applied on $N_{+}=a N_{V}$ vertices whereas $-F^{e x t}$ is applied on $N_{-}=a N_{V}$ vertices along the principal axis of inertia ${ }^{3}$ (refer to Figure 2) [1, 34]. $N_{+}$and $N_{-}$are the vertices that locate within the circular region of radius $d_{C} / 2$ on the initial spherical geometry, and from the 2 vertices $\left(i_{X_{\max }}\right.$ and $i_{X_{\min }}$ ) on the furthest ends of the equilibrium cell shape along the principal axis of inertia ${ }^{3} \cdot d_{C}$ is the contact diameter between the cell membrane and the attached silica beads. The vertex fraction $a$ corresponds to $d_{C}$ and is given by $a=\pi d_{C}^{2} /\left(4 A_{0}\right)$. Therefore, $f_{i}^{e x t}$ is applied on $i^{\text {th }}$ vertex, such that;

$$
f_{i}^{\text {ext }}=\left\{\begin{array}{cc}
F^{\text {ext }} / N_{+}, & i \in N_{+} \\
-F^{\text {ext }} / N_{-}, & i \in N_{-} \\
0, & i \notin\left(N_{+} \cup N_{-}\right)
\end{array}\right.
$$

(a)
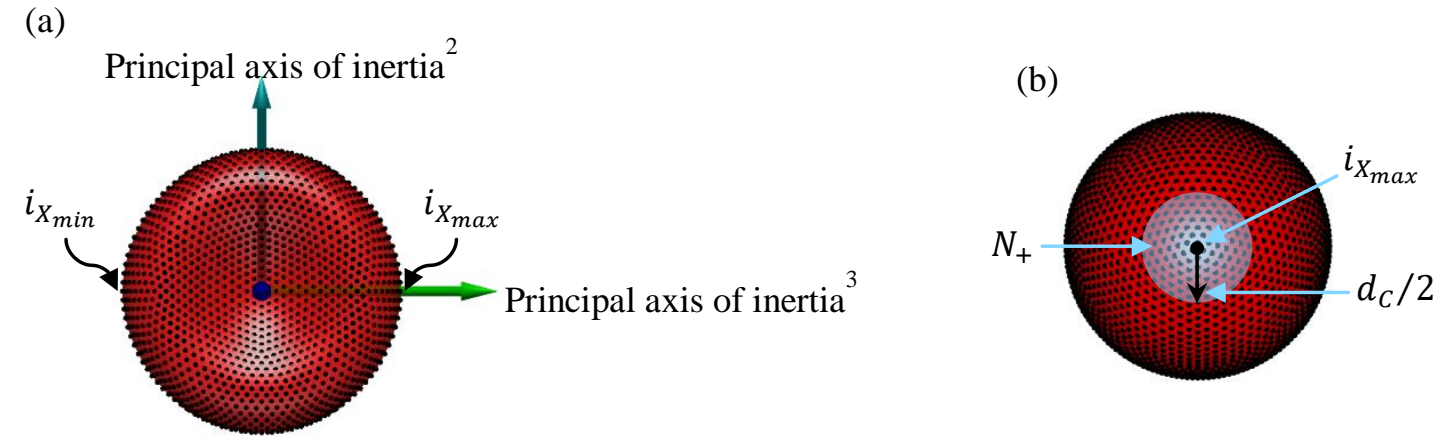

(c)

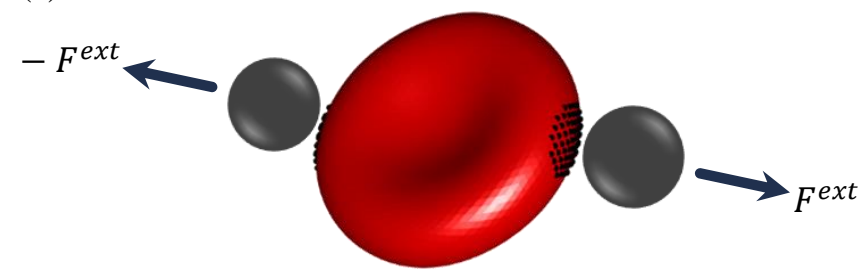

Figure 2 - Optical tweezers stretching implementation on discocyte cell; (a) identification of $i_{X_{\max }}$ and $i_{X_{\min }}$ vertices, (b) identification of $N_{+}$vertices, and (c) contact region between RBC membrane and binding silica beads

$F^{e x t}$ is gradually applied on the cell via regular force increments of $\Delta F^{e x t}$ where the cell is provided with sufficient time to converge to the equilibrium stretched state after each force increment. The equilibrium stretched cell state is determined at equivalent $A_{0}, A_{k, 0}, V_{0}, \Delta A_{0}$ and $C_{0}$ for stomatocyte, discocyte and echinocyte cell shapes and at corresponding $F^{\text {ext }}$. The resultant force and motion of $i^{\text {th }}$ membrane vertex is determined according to Equations 11 and 12 for $F^{\text {ext }}$ external force, and the equilibrium cell state is determined. 


\subsection{Validation of CG-RBC Membrane Model Predicted Discocyte Deformation Behaviour}

The optical tweezers stretching deformation of the equilibrium discocyte cell is investigated at equivalent reference conditions and model parameters. Analogous to experimental optical tweezers stretching experiments by Suresh et al. [66], $F^{\text {ext }}$ is applied on the cell such that $0 \leq F^{\text {ext }} \leq 200.0$ pN. $d_{C}$ is approximated as $2.0 \mu \mathrm{m}[1,2,9,34,66]$, and accordingly $F^{\text {ext }}$ is applied on $a=0.02$ membrane vertices in regular increments of $\Delta F^{e x t}=10.0 \mathrm{pN}$. The evolution of axial diameter $\left(D_{A}\right)$ (measured along the principal axis of inertia ${ }^{3}$ ) and transverse diameter $\left(D_{T}\right)$ (measured along the principal axis of inertia ${ }^{2}$ ) of the equilibrium stretched cell state at $F^{\text {ext }}$, are then compared against experimental observations by Suresh et al. [66]. Refer to Figure 3 for a graphical representation of $D_{A}$ and $D_{T}$.

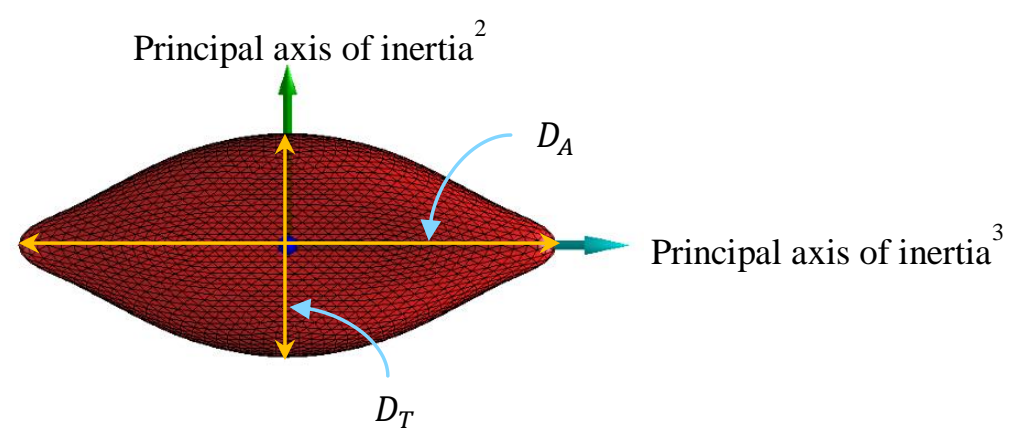

Figure 3 - Measurement of axial $\left(D_{A}\right)$ and transverse $\left(D_{T}\right)$ diameters of a RBC at equilibrium cell stretched state

It can be observed (refer to Figure 4) that at the model parameters given in Table 1, the deformation behaviour of the discocyte indicates considerable stiffness against the analogous experimental observations. The maximum deviation $\left(\varepsilon_{\text {OTS }}\right)$ between numerically predicted and experimentally observed $D_{A}$ is $19.679 \%$ in this scenario, and significantly high. The discocyte deformation behaviour is highly sensitive to $\mu_{0}, x_{0}$ and $k_{A D}$ (refer to section 3.4), and therefore the presence of $E_{\text {Area-difference }}$ has introduced additional stiffness to the cell during its attempt to maintain the corresponding $\Delta A_{0, \text { Discocyte }}$. Therefore, $k_{A D}$ is amended such that the discocyte cell behaviour better agrees with the experimental observations $\left(\varepsilon_{\text {OTS }}<10.0 \%\right)$, and stomatocyte, discocyte and echinocyte cell morphologies can also be successfully predicted at this updated value of $k_{A D}$. At $k_{A D}=7.5 \times 10^{-18}$ $\mathrm{Nm}$, the discocyte deformation behaviour agrees reasonably with the experimental observations $\left(\varepsilon_{O T S}\right.$ $=8.191 \%$ ), and remains within the experimental error bars. The disagreement in the $D_{T}$ may be partially due to experimental errors arising from the fact that the optical tweezers stretching measurements have been performed from a single observation angle [34, 36]. RBC undergoing stretching may rotate on the plane perpendicular to the initial principal axis of inertia ${ }^{3}$, and therefore measurements from a single observation angle may lead to deviations from actual $D_{T}$. The numerically estimated $D_{T}$ is measured 
along the principal axis of inertia ${ }^{2}$ of the equilibrium cell stretched state, and therefore gives improved accuracy.

In addition, similar equilibrium $\mathrm{RBC}$ morphologies are also achieved at the corresponding $\Delta A_{0, \text { Stomatocyte }}, \Delta A_{0, \text { Discocyte }}$ and $\Delta A_{0, \text { Echinocyte }}$ at $k_{A D}=7.5 \times 10^{-18} \mathrm{Nm}$ (refer to Figure 5). The BCM-based membrane energy approach can predict stable vesicle shapes only if the ratio of $k_{A D} / \kappa$ is above the critical value at $v$ of the vesicle [49,67, 68]. $v$ is defined as the cell volume relative to the sphere having equivalent cell surface area, and equals to 0.6 for the present $A_{0}$ and $V_{0}$. The critical ratio of $k_{A D} / \kappa$ at $v=0.6$ is approximately $3[49,67]$, whereas for the present consideration $k_{A D} / \kappa$ is 30 , a value well above the critical ratio. Therefore, the CG-RBC membrane model is capable of predicting stable vesicle shapes at $k_{A D}=7.5 \times 10^{-18} \mathrm{Nm}$, and it is reasonable to adopt CG-RBC membrane model at $k_{A D}=7.5 \times 10^{-18} \mathrm{Nm}$ to investigate the deformation behaviour of stomatocyte, discocyte and echinocyte morphologies undergoing optical tweezers stretching.

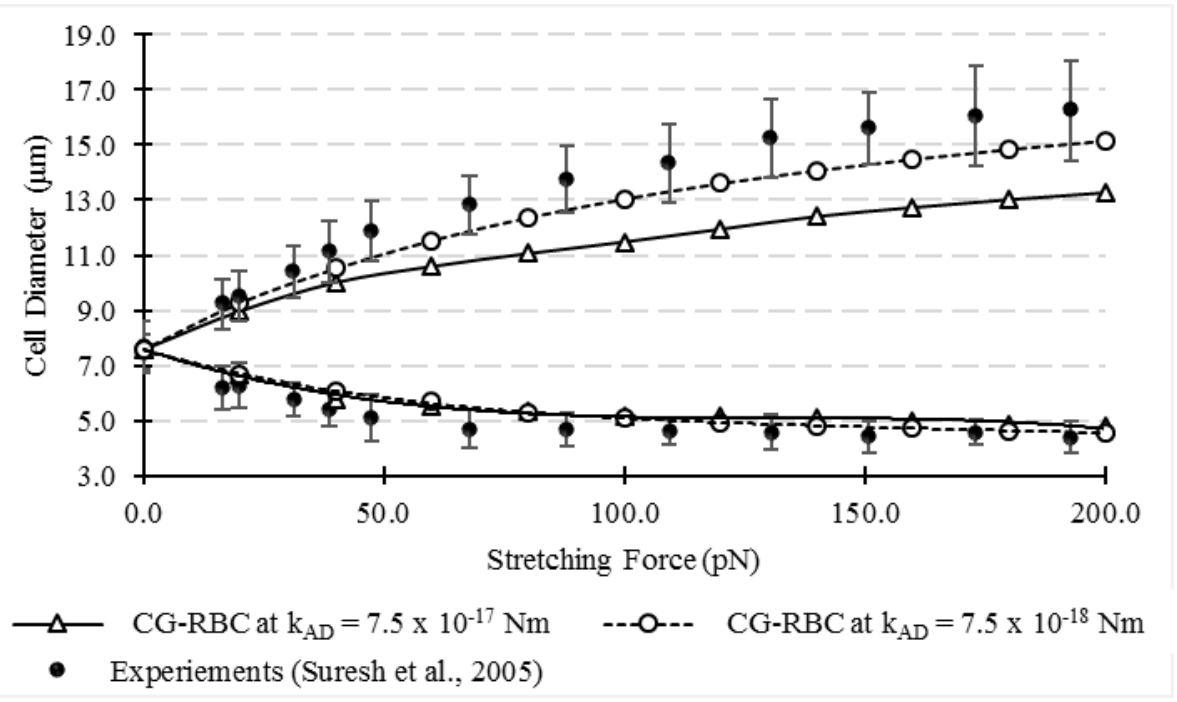

Figure 4 - Comparison between CG-RBC membrane model predicted deformation behaviour at $k_{A D}=7.5 \mathrm{x}$ $10^{-17} \mathrm{Nm}$ and $k_{A D}=7.5 \times 10^{-18} \mathrm{Nm}$, versus experimentally observed [66] deformation behaviour of discocyte cell undergoing optical tweezers stretching. The curves in the top represent the evolution of axial diameter $\left(D_{A}\right)$ whereas the curves in the bottom represent the evolution of transverse diameter $\left(D_{T}\right)$.

(a)

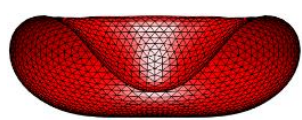

(b)

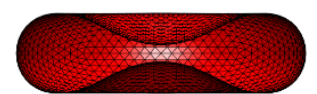

(c)

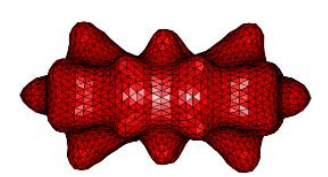

Figure 5 - The CG-RBC membrane model predicted stomatocyte, discocyte and echinocyte morphologies at $k_{A D}=7.5 \times 10^{-18} \mathrm{Nm}$. 


\section{Results and Discussion}

\subsection{Evolution of Cell Shape and Membrane Free Energy of Stomatocyte, Discocyte and Echinocyte Morphologies}

The shape deformation behaviour of stomatocyte, discocyte and echinocyte morphologies at equilibrium stretched cell state are studied. The membrane shear modulus $\left(\mu_{0, \text { Echinocyte }}\right)$ and bending modulus ( $\kappa_{\text {Echinocyte }}$ ) of echinocyte shape are adjusted to $7.17 \mu \mathrm{Nm}^{-1}$ and $4.36 \times 10^{-19} \mathrm{Nm}$, respectively in order to adopt the stiffer nature of the echinocyte cell shape [15, 25, 26, 30, 40, 69-71]. Correspondingly, $k_{A D}$ and $k_{C}$ for echinocyte shape ( $k_{A D, E c h i n o c y t e}$ and $\left.k_{C, E c h i n o c y t e}\right)$ are also adjusted to $1.31 \times 10^{-16} \mathrm{Nm}$ and $4.36 \times 10^{-17} \mathrm{Nm}$, respectively to maintain equivalent $k_{A D} / \kappa(=30.0)$ and $k_{C} / \kappa$ $(=100.0)$ for all cell types. Figure 6 compares the cell shape evolution from initial equilibrium (at $F^{\text {ext }}$ $=0 \mathrm{pN})$ cell state to equilibrium stretched cell states at $F^{\text {ext }}=60.0 \mathrm{pN}$ and $F^{\text {ext }}=200.0 \mathrm{pN}$.

It can be observed that the morphology characteristics have been preserved for all cell types; stomatocyte, discocyte and echinocyte during stretching. However, the depths of concavity of stomatocyte and biconcavity of discocyte, and the spicule height of echinocyte shape, have reduced with the increment of $F^{\text {ext }}$. During the stretching of the cell, there are two competitive membrane deformation effects: one caused by the cell morphology and the other caused by the stretching forces. At small stretching forces, it is less favourable for the membrane to be moved from the concavity of stomatocyte, biconcavity of discocyte and spicules of echinocyte into the stretched part of the membrane. Therefore, the spectrin links are relatively compressed in the region away from the cell membrane-bead contact, whereas the spectrin links are elongated in the region near the cell membranebead contact. This results in extra resistance of the cell at small stretching forces. However, at large stretching forces, the cell membrane moves from the concavity of stomatocyte, biconcavity of discocyte and spicules of echinocyte into the stretched part of the membrane (refer to Figures 10 and 11). The gradually increasing cell elongation along the principal axis of inertia ${ }^{3}$ with the increment of $F^{\text {ext }}$, contributes to $\Delta A$ and $C$ to a greater extent. Therefore, the equilibrium stretched cell state achieves the specified $\Delta A_{0}$ and $C_{0}$ at either lesser concavity depth or lesser spicule height. In addition, the discocyte shape indicates asymmetric biconcavity at higher $F^{\text {ext }}$. Li et al. [72] have also observed asymmetric biconcavity in the resultant equilibrium stretched state of a discocyte at $F^{\text {ext }}=100.0 \mathrm{pN}$ with respect to the choice of bending energy expression adopted (refer to Figure 7 and Equations 4 and 15 in [72]). However, the observed influence of the choice of bending energy expression on discocyte stretching response, is small [72].

(a) Stomatocyte 

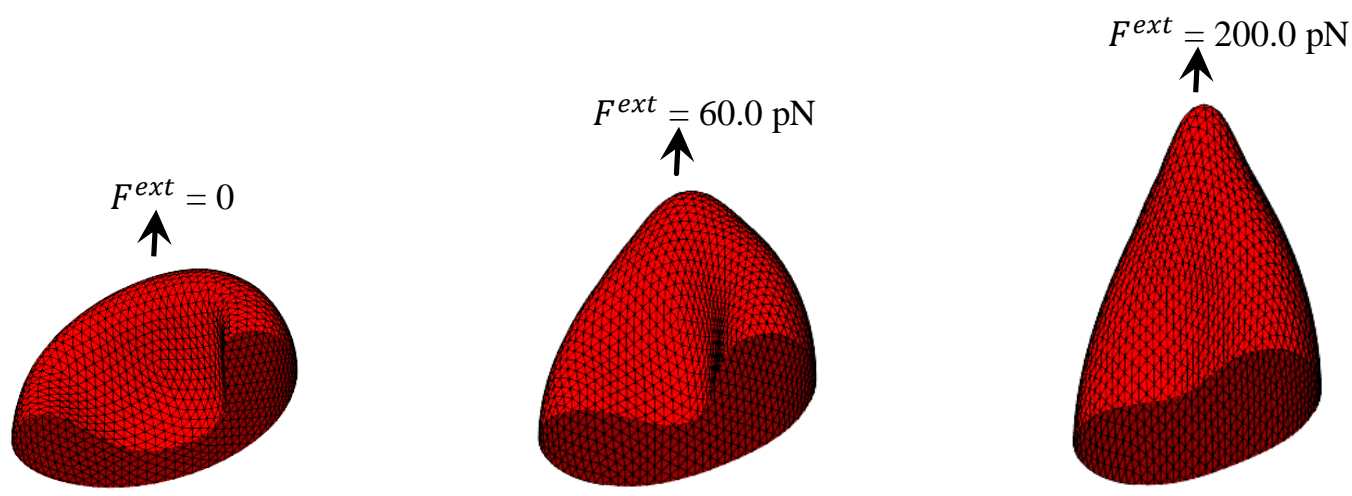

(b) Discocyte
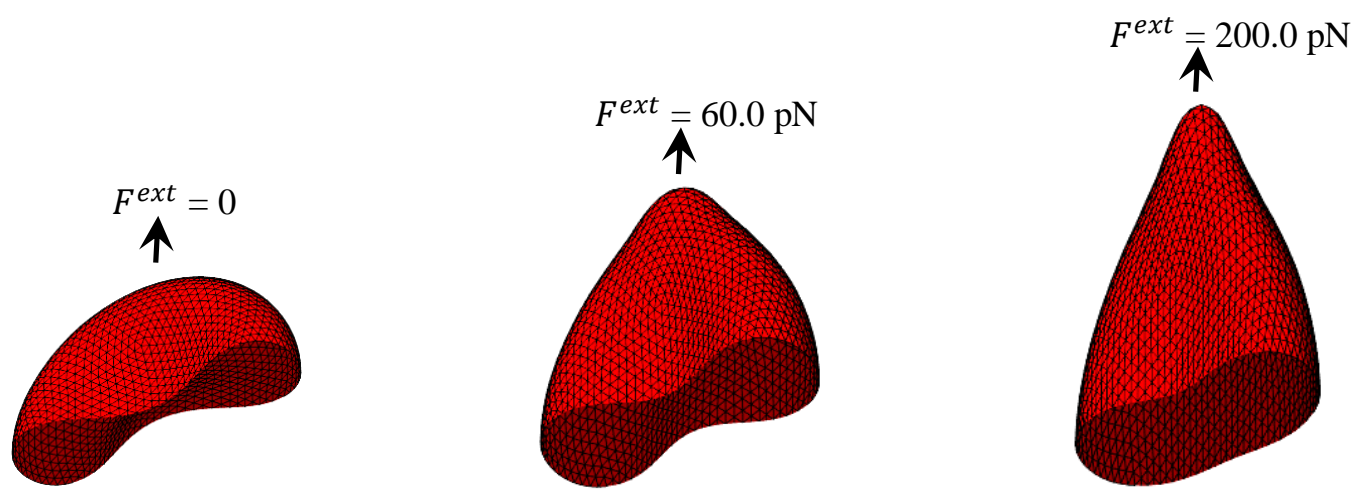

(c) Echinocyte
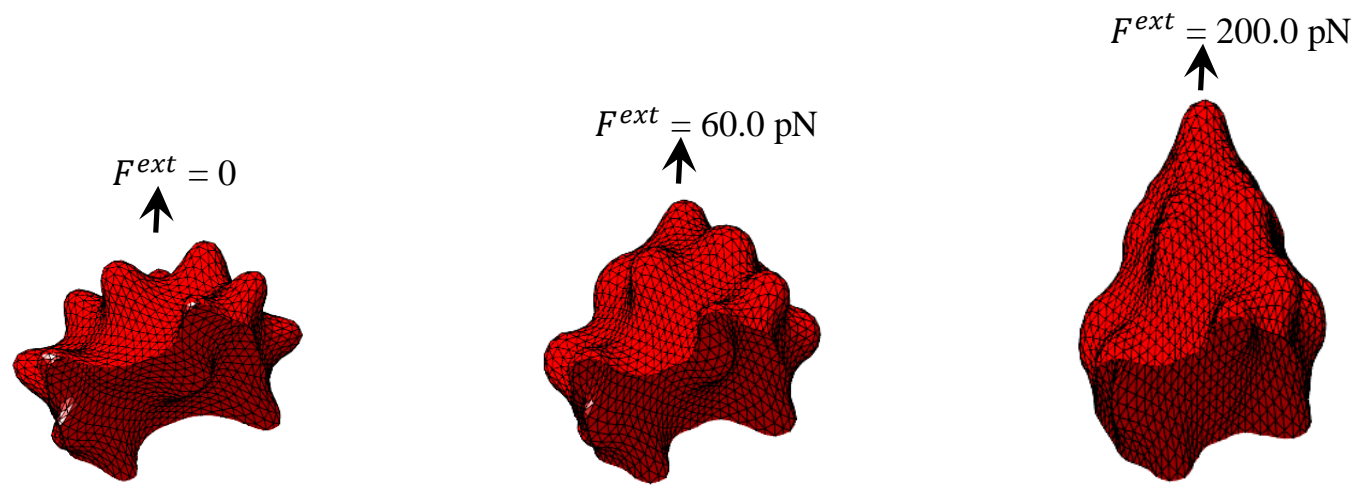

Figure 6 - Shape deformation of (a) stomatocyte, (b) discocyte and (c) echinocyte RBC morphologies at $F^{\text {ext }}=0,60.0 \mathrm{pN}$ and $200.0 \mathrm{pN}$ stretching forces

The evolution of membrane free energy in response to the applied $F^{\text {ext }}$ (refer to Figure 7) confirms the increased struggle to maintain the equilibrium cell shape against applied external stretching forces. For clarity, only the evolution of energy components of discocyte at its equilibrium stretched cell state are presented in Figure 7, and are expressed in the form of logarithmic values of base 10. $E$ is higher for the echinocyte, and can be attributed to the higher $\mu_{0}$ and $\kappa$ of echinocyte shape than that for stomatocyte and discocyte. $E_{\text {Stretching }}$ is the dominant contributor to $E$, and increases with $F^{\text {ext }}$ for all cell shapes. The influence of $E_{\text {Area-difference }}$ for stomatocyte and discocyte cells being notable at high $F^{e x t}$, indicates the increased struggle to maintain $\Delta A_{0}$ at high $F^{\text {ext }}$. However, echinocyte shape is mostly 
influenced by $E_{\text {Stretching }}$ in response to $F^{e x t}$ and by $E_{\text {Bending }}$ in response to its spiculated nature. The negligible contribution of $E_{\text {Surface-area }}, E_{\text {Volume }}$ and $E_{\text {Total-curvature }}$ to $E$ at equilibrium cell stretched state, indicates that the cells have achieved $A_{0}, A_{k, 0}, V_{0}$ and $C_{0}$ reference conditions at the equilibrium. However, the cell requires more effort to maintain its reference conditions in the presence of global stretching deformation that induce shearing to cell membrane. The minimal influence $E_{V o l u m e}$ indicates the incompressibility of the cell, and the effect of the optical tweezers stretching forces are therefore dissipated onto the cell membrane.

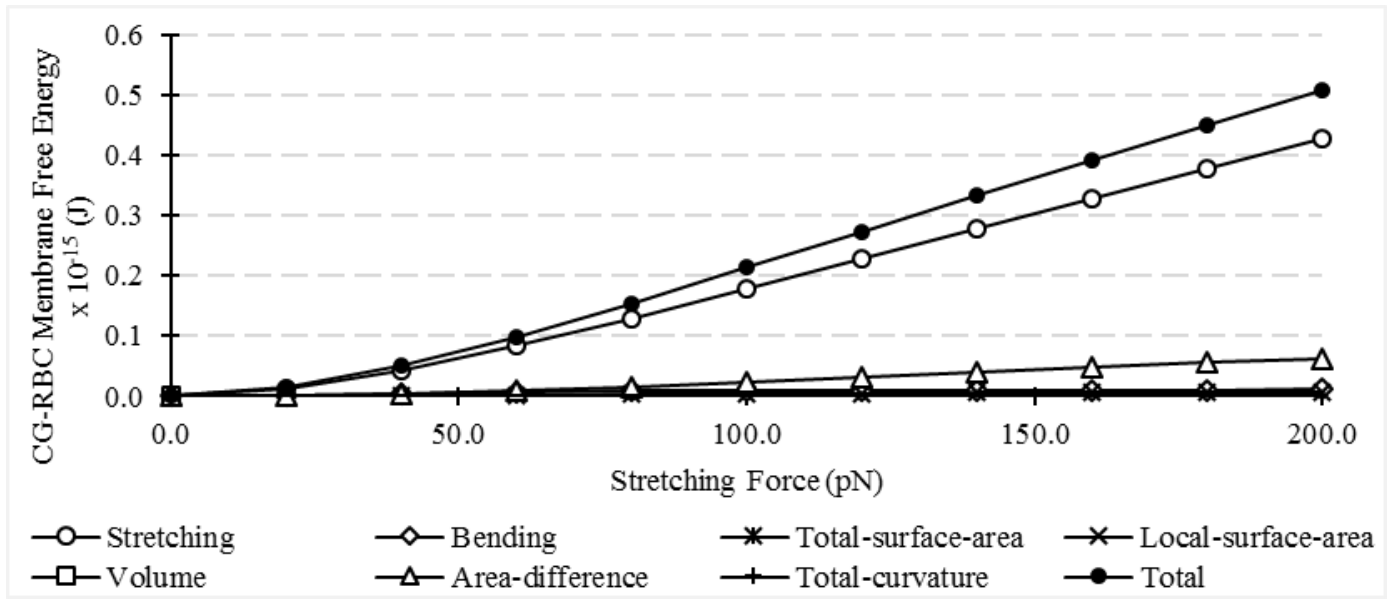

Figure 7 - Evolution of RBC membrane free energy of discocyte morphology in response to $F^{\text {ext }}$ at equilibrium stretched cell states

\subsection{Change in Cell Diameters and Elongation Index of Stomatocyte, Discocyte and Echinocyte Morphologies}

The stretching response of stomatocyte, discocyte and echinocyte cells at $F^{\text {ext }}$ up to a maximum value of $\sim 200.0 \mathrm{pN}$, is presented in Figure 8. $D_{A}$ and $D_{T}$ of stomatocyte and echinocyte morphologies are estimated along the principal axis of inertia ${ }^{3}$ and the principal axis of inertia ${ }^{2}$, respectively, and are analogous to the estimation of $D_{A}$ and $D_{T}$ of discocyte morphology. Overall, $D_{A}$ increases while $D_{T}$ decreases with increasing $F^{\text {ext }}$. The percentage change in $D_{A}$ and $D_{T}$ at $F^{\text {ext }}$ is used to compare the stretching response of these cell shapes in order to exclude the influence of differences in initial cell dimensions. The percentage change in $D_{A}$ is estimated as follows,

$$
\text { Change in } D_{A}=\frac{D_{A}-D_{A, 0}}{D_{A, 0}} \times 100 \%
$$

where, $D_{A, 0}$ is the axial cell diameter of stomatocyte, discocyte and echinocyte cell shapes at $F^{e x t}=0$. Similarly, the percentage change in $D_{T}$ is estimated at $F^{\text {ext }}$ with respect to the initial transverse cell diameter $\left(D_{T, 0}\right)$ corresponding to $F^{\text {ext }}=0$. 


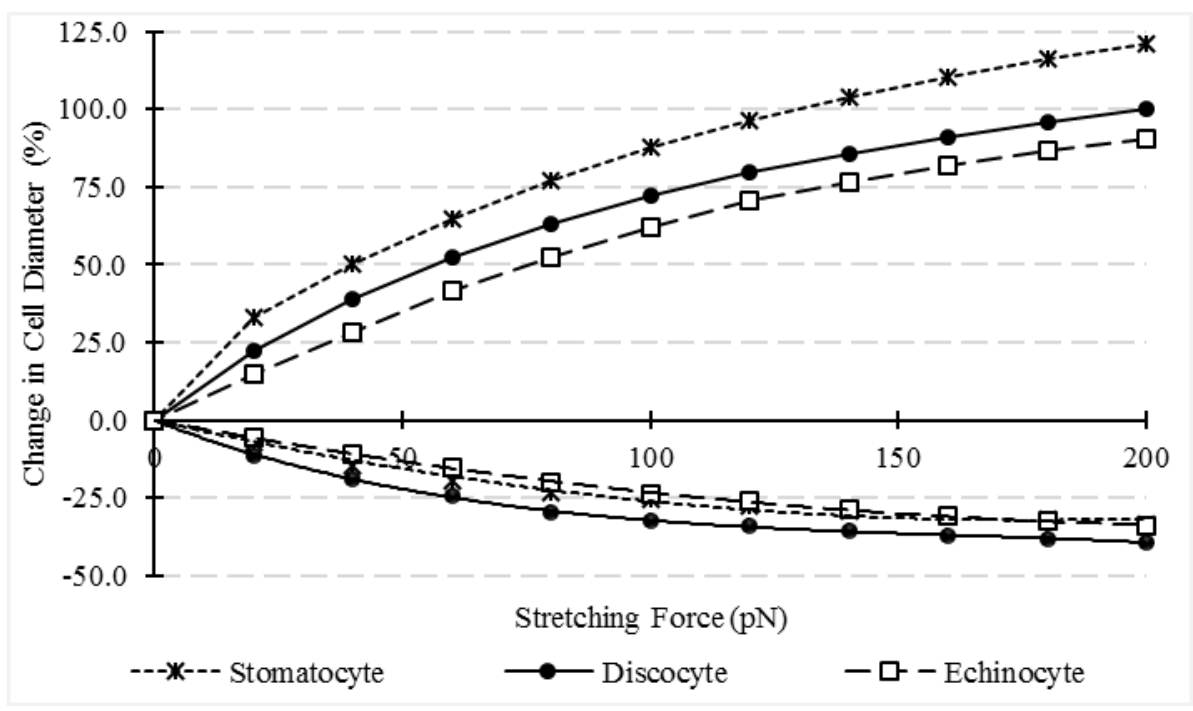

Figure 8 - Comparison of the deformation behaviour of stomatocyte, discocyte and echinocyte cells undergoing optical tweezers stretching. The curves in the top represent the change in $D_{A}$ whereas the curves in the bottom represent the change in $D_{T}$.

It can be observed that the stomatocyte indicates the highest change in $D_{A}$ at all $F^{\text {ext }}$ whereas the lowest is for echinocyte shape. However, discocyte indicates the highest change in $D_{T}$ at all $F^{\text {ext }}$. Stomatocyte indicates a higher change in $D_{T}$ than that of echinocyte shape at low to moderate $F^{\text {ext }}$ whereas echinocyte indicates higher change in $D_{T}$ than that of stomatocyte at high $F^{\text {ext }}$. The stomatocyte retains it characteristic reference conditions and stretches along the axial direction of stretching and reduces its concavity depth at a small change in $D_{T}$ (refer to Figure 6). Therefore, the equilibrium stretched cell state of stomatocyte indicates a large change in $D_{A}$ and a small change in $D_{T}$ in the presence of $F^{\text {ext }}$. Echinocyte indicates a lower change in both $D_{A}$ and $D_{T}$ due to its high $\mu_{0}$. The increasing cell bulging along the axial direction of stretching, assists the echinocyte cell to achieve $\Delta A_{0}$ and $C_{0}$. Therefore, the height of membrane spicules on echinocyte progressively reduces with the increase in $F^{\text {ext }}$ (refer to Figure 6), and leads to an increased change in $D_{T}$ at high $F^{\text {ext }}$.

The RBC deformability measure, elongation index $(E I)$ is adopted to investigate the deformability of stomatocyte, discocyte and echinocyte cell shapes undergoing optical tweezers stretching deformation. The larger the value of $E I$ at $F^{e x t}$, the more deformable the cell is. EI is estimated at equilibrium cell stretched state based on $D_{A}$ and $D_{T}$, such that [5, 29];

$$
E I=\frac{D_{A}-D_{T}}{D_{A}+D_{T}}
$$

The evolution of $E I$ values of stomatocyte, discocyte and echinocyte cell shapes, is presented in Figure 9. EI value for every cell shape increases with increasing $F^{\text {ext }}$. The deformation behaviour of RBCs undergoing optical tweezers stretching is primarily due to the cytoskeletal stretching response [66, 72], and therefore the evolution of $E I$ is primarily led by cytoskeletal stretching characteristics. The decreasing gradient of $E I$ versus $F^{\text {ext }}$, suggests reduced stretchability of cells at increasing $F^{\text {ext }}$. This 
stretching response can be attributed to the influence of spectrin link extensibility $\left(x_{0}\right)$, which limits the maximum extensibility of spectrin links in the presence of $F^{e x t}$. Furthermore, the approximately similar values of $E I$ of all cell shapes at higher $F^{e x t}$ indicate the influence of $x_{0}$ such that the stretchability of the cell is inhibited. The approximately similar $E I$ value of echinocyte to that of discocyte at low $F^{\text {ext }}$ can be attributed to the initial echinocyte shape, which is a slightly elongated cell. However, echinocyte indicates the lowest deformability and agrees with the experimentally observed stiffened nature of echinocytes [15, 23, 32, 40, 73, 74].

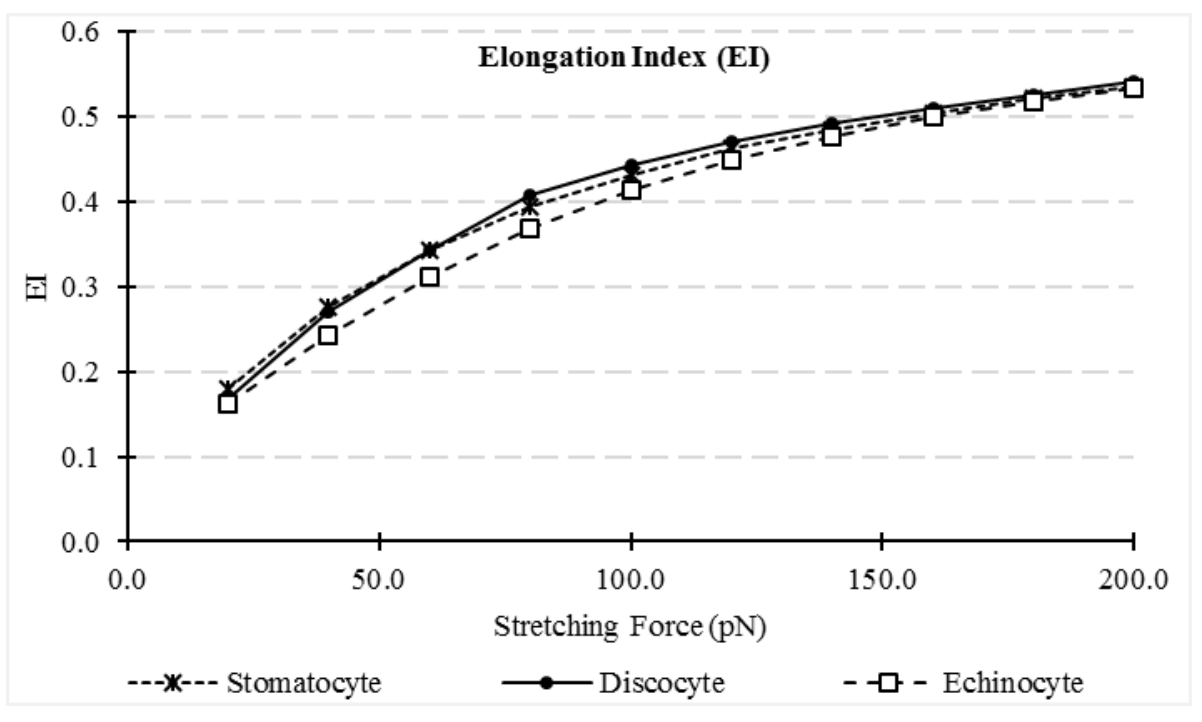

Figure 9 - Comparison of the elongation indices (EI) of stomatocyte, discocyte and echinocyte cells undergoing optical tweezers stretching

\subsection{Extension of Spectrin Links $\left(e_{j}\right)$ of Stomatocyte, Discocyte and Echinocyte Morphologies}

The cytoskeletal spectrin network is the primary contributor for RBC stretching deformation under optical tweezers stretching forces. Therefore, the extension of CG spectrin links is investigated at several $F^{\text {ext }}\left(F^{\text {ext }}=0,60.0\right.$ and $\left.200.0 \mathrm{pN}\right)$. The extension of the $j^{t h}$ spectrin link $\left(e_{j}\right)$ with respect to its equilibrium spectrin link length at cytoskeletal reference state $\left(l_{0, j}\right)$, is defined as follows;

$$
e_{j}=\frac{l_{j}-l_{0, j}}{l_{0, j}} \times 100 \%
$$

where, $l_{j}$ is the length of $j^{\text {th }}$ spectrin link at equilibrium stretched cell state. $e_{j}$ indicates the level of folding and unfolding of spectrin links, and comparable to the strain along the axial direction of spectrin links. Any $e_{j}$ value less than zero indicates contraction of spectrin links. The comparison of $e_{j}$ for stomatocyte, discocyte and echinocyte cell shapes at $F^{e x t}=0,60.0$ and $200.0 \mathrm{pN}$ is presented in Figure 10 whereas Figure 11 compares the percentage number of spectrin links on CG-RBC membrane having $e_{j}$ within a specific range of extension. It can be observed that the discocyte is composed of spectrin links of $e_{j}$ very close to its $l_{0, j}$ whereas the stomatocyte cell shape has spectrin links of moderately extended and contracted on its concavity. However, the echinocyte cell is composed of spectrin links 
of which $e_{j}$ is varied within a wide range of extension and contraction. It can be observed that the tips of the spicules and valleys between the spicules on echinocyte cell are composed of spectrin links of negligible extension or contraction. The spectrin links that are parallel to the contour lines on membrane spicules indicate low to moderate contraction, whereas the spectrin links that cross the contour lines on membrane spicules indicate low to the moderate extension.

In general, the spectrin links along the axial direction of $F^{\text {ext }}$ indicate extension, whereas the spectrin links along the transverse direction of $F^{\text {ext }}$ indicate contraction due to optical tweezers stretching forces. The distribution of the fraction of spectrin links having specific $e_{j}$ is similar for stomatocyte and discocyte cell shapes, whereas echinocyte cell shape indicates a notable difference from that of stomatocyte and discocyte. Low to moderate levels of extension and contraction of spectrin links are observed for all three cell shapes at moderate $F^{\text {ext }}$. However, the extension and contraction of spectrin links at higher $F^{\text {ext }}$, are distributed in a wide range of $e_{j}$. The major fraction of spectrin links of stomatocyte $(=24.24 \%)$ and discocyte $(=24.94 \%)$ cells at $F^{e x t}=200.0 \mathrm{pN}$ is composed of spectrin links having $e_{j}>50.0 \%$ to compensate the externally applied stretching forces. Even though, the fraction of echinocyte spectrin links having higher $e_{j}$ is notable at $F^{\text {ext }}=200.0 \mathrm{pN}$, the fraction of spectrin links having $e_{j}>50.0 \%$ is only $13.85 \%$. This behaviour can be attributed to the high $\mu_{0}$ of echinocyte cell, which affect the stretchability of the cell. The contribution from cytoskeletal spectrin network is essential to observe echinocytosis on $\operatorname{RBC}$ membrane $[18,22,54,56,58,60]$. Therefore, the fraction of echinocyte spectrin links having specific $e_{j}$ is distributed in a wide range $e_{j}$ values such that the spicules on the RBC membrane are formed. The distribution of $e_{j}$ indicates the changes in spectrin link density during stretching deformation, and agrees with previous studies [3]. The membrane mechanical deformation causes a lateral redistribution of the membrane such that the density is increased in regions where the stretching deformation is high and vice versa [3].

(a) Stomatocyte
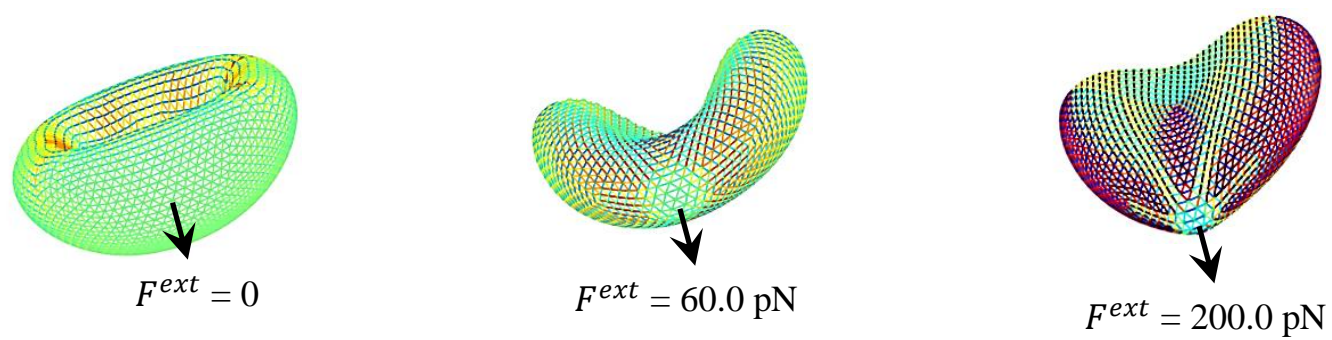

(b) Discocyte 

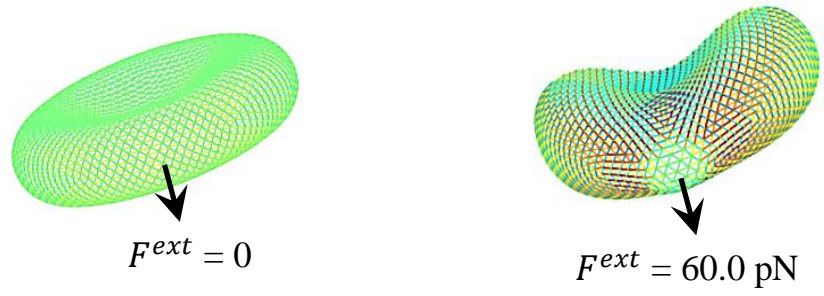

(c) Echinocyte

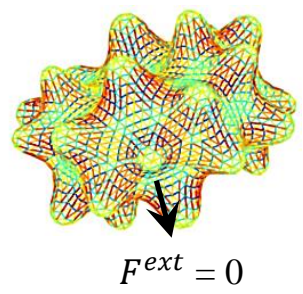

$$
F^{\text {ext }}=0
$$

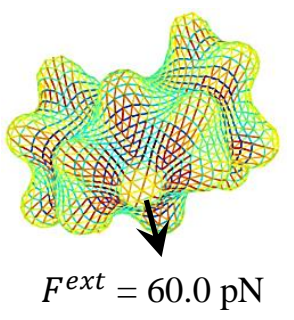

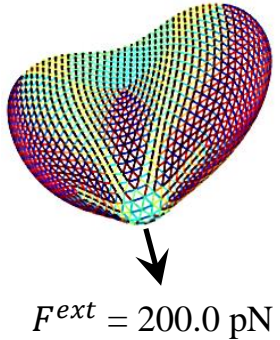

$F^{e x t}=200.0 \mathrm{pN}$

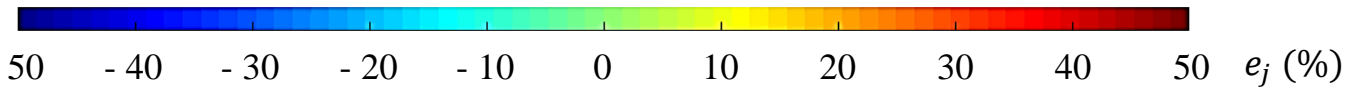

Figure 10 - Comparison of the spectrin link extension, $e_{j}(\%)$ on spectrin links of (a) stomatocyte, (b) discocyte and (c) echinocyte cells at $F^{e x t}=0,60.0$ and $200.0 \mathrm{pN}$ optical tweezers stretching forces. The fullcell view is presented at $F^{e x t}=0$ whereas half-cell view is presented for $F^{e x t}=60.0$ and $200.0 \mathrm{pN}$ instances. 

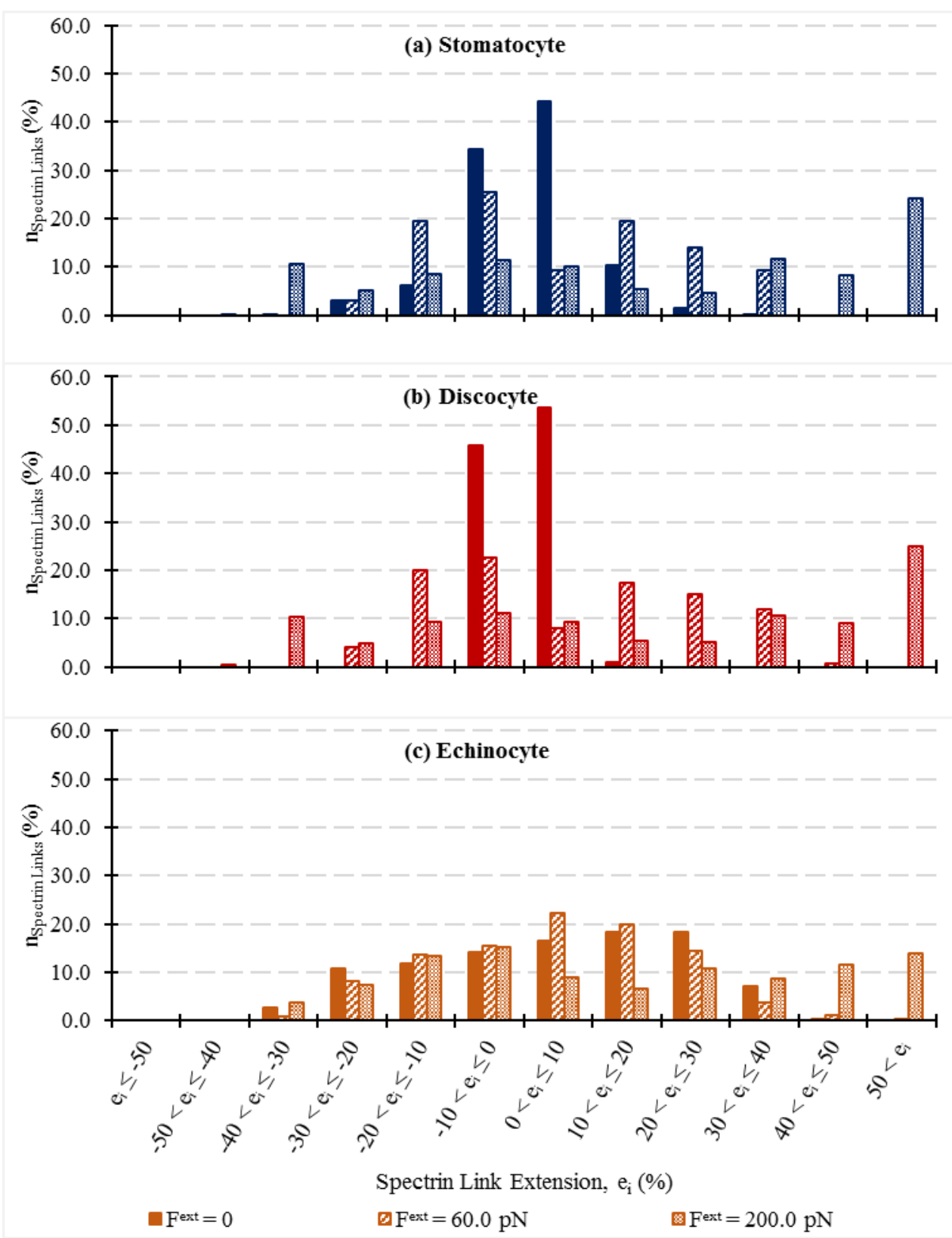

Figure 11 - Comparison of the number of spectrin links having specific spectrin link extension, $e_{i}(\%)$ of (a) stomatocyte, (b) discocyte and (c) echinocyte cells at $F^{e x t}=0,60.0$ and $200.0 \mathrm{pN}$ optical tweezers stretching forces

\subsection{Effect of Change in CG-RBC Membrane Model Parameters on Stomatocyte, Discocyte and Echinocyte Deformation Behaviour}

Parametric analyses are conducted to investigate the influence of RBC membrane shear modulus $(\mu)$, bending modulus $(\kappa)$, spectrin link extensibility $(x)$ and RBC membrane-bead contact diameter $\left(d_{C}^{\prime}\right)$ on deformation behaviour of stomatocyte, discocyte and echinocyte cell shapes undergoing optical tweezers stretching deformation. The improved understanding on the influence of model parameters and their reflectance on RBC deformability, improves the applicability of optical tweezers stretching 
experiments to investigate RBC functionality under different pathophysiological conditions [5, 34, 66]. The above-mentioned CG-RBC membrane model parameters are varied in isolation where equivalent model parameters as mentioned in section 3.1 are adopted as the baseline model parameters. The evolution of $E I$ of stomatocyte, discocyte and echinocyte is then studied for $F^{\text {ext }}(\mathrm{pN})=[0,200]$.

The influence of $\mu$ on stretching behaviour of RBCs having varied morphology is investigated. Several $\mu$ values such that $\mu\left(\mu \mathrm{Nm}^{-1}\right)=\left[0.75 \mu_{0}, 1.5 \mu_{0}\right]$. Figure 12 (a) represents the comparison of $E I$ at $\mu=$ $0.75 \mu_{0}, 1.0 \mu_{0}$ and $1.5 \mu_{0}$ for discocyte cell shape. For clarity, only the evolution of $E I$ of discocyte at varying shear modulus $(\mu)$, spectrin link extensibility $(x)$, membrane bending modulus $(\kappa)$, and RBC membrane-bead contact diameter $\left(d_{C}^{\prime}\right)$, is presented in Figure 12, and stomatocyte and echinocyte cells indicate similar behaviour as well. It can be observed that the change in $\mu$ has an inverse impact on the $E I$ of every cell shape. The increase in $\mu$ increases the effort required to stretch the cells, and therefore the observed $E I$ reduces. Similarly, the influence of $x$ is investigated at several $x$ values such that $x=$ $\left[0.75 x_{0}, 1.5 x_{0}\right] . x$ is the ratio between the equilibrium length of $j^{\text {th }}$ spectrin link $\left(l_{0, j}\right)$ at cytoskeletal reference state to its maximum spectrin link length $l_{\max , j}$. Therefore, a lower value of $x$ indicates higher spectrin link extensibility whereas a higher value of $x$ indicates lower spectrin link extensibility. Several values of spectrin link extensibility have been adopted in different CG-spectrin level RBC membrane studies [1, 22, 34, 72, 75-78], and the present value of $x_{0}=0.45$ is equal to the value adopted by Fedosov et al. [34] in their CG-spectrin level model development. All the other model parameters are equal to the corresponding model parameters as in section 3.1. It can be observed (refer to Figure 12 (b)) that the change in $x$ also has an inverse impact on $E I$ of RBC cell shapes. However, the influence of varied $x$ is notable at moderate to high $F^{e x t}$. This behaviour corresponds to the decreased maximum spectrin link extensibility at higher $x$, which then leads to inhibited global cell stretchability. 

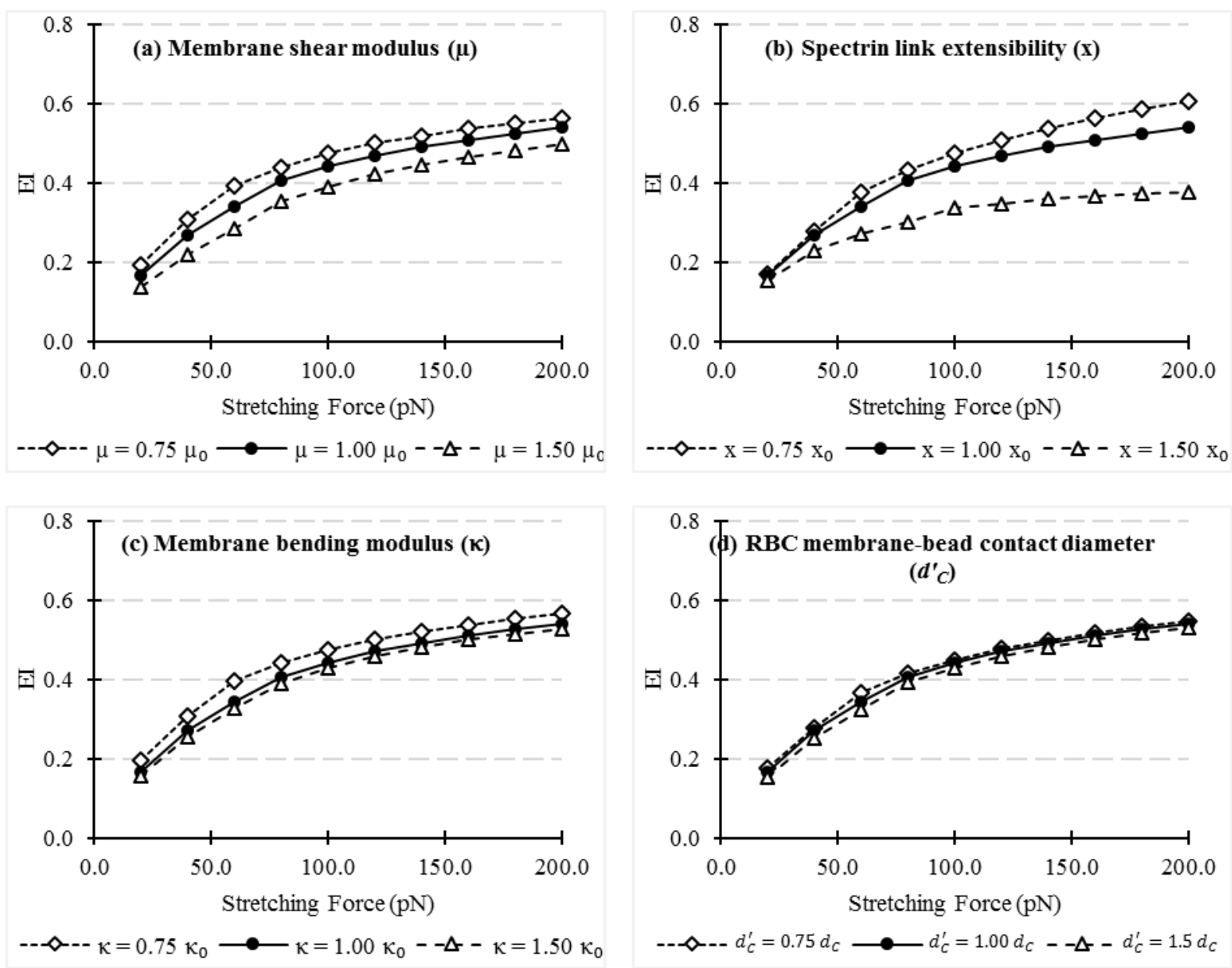

Figure 12 - Evolution of the elongation indices $(E I)$ of the discocyte cell undergoing optical tweezers stretching at varying (a) shear modulus $(\mu)$, (b) spectrin link extensibility $(x)$, (c) membrane bending modulus $(\kappa)$, and (d) RBC membrane-bead contact diameter $\left(d_{C}^{\prime}\right)$

Moreover, the CG-RBC membrane model is adopted to investigate the membrane properties of an echinocyte cell undergoing optical tweezers stretching deformation. The echinocyte being less deformable than a healthy $\mathrm{RBC}[15,69,70]$, and the RBC stretching response being mainly governed by the cytoskeletal stretching characteristics, the limits of membrane shear modulus $\left(\mu_{0, \text { Echinocyte }}\right)$ and spectrin link extensibility $\left(x_{0, \text { Echinocyte }}\right)$ of echinocyte cell shape are estimated such that the deformability of echinocyte decreases more than that of a discocyte. Several $\mu$ values such that $\mu\left(\mu \mathrm{Nm}^{-}\right.$ $\left.{ }^{1}\right)=\left[1.0 \mu_{0, \text { Discocyte }}, 3.0 \mu_{0, \text { Discocyte }}\right]$ are achieved at 0.25 multiplications of $\mu_{0, \text { Discocyte }}\left(\mu_{0, \text { Discocyte }}=\right.$ $\left.4.0 \mu \mathrm{Nm}^{-1}\right)$ at constant $\kappa\left(\kappa=2.5 \times 10^{-19} \mathrm{Nm}\right)$, where $\mu_{0, \text { Discocyte }}$ and $\kappa$ are the cytoskeletal shear modulus and membrane bending modulus of a healthy discocyte. All the other model parameters are equal to the corresponding model parameters as in section 3.1. The stretching deformation of echinocyte cell shape is studied for $F^{e x t}(\mathrm{pN})=[0,200]$ at above-mentioned $\mu$ values, and the evolution of $E I$ is compared against that of discocyte $E I$ at $\mu_{0}$. Figure 13 (a) represents the comparison of $E I$ of the echinocyte cell at $\mu=1.5 \mu_{0, \text { Discocyte }}, 1.75 \mu_{0, \text { Discocyte }}$ and $2.0 \mu_{0, \text { Discocyte }}$ against $E I$ of discocyte at $\mu_{0, \text { Discocyte. At }} \mu=2.0 \mu_{0, \text { Discocyte }}$, echinocyte indicates decreased deformability than that of the 
discocyte for the complete range of $F^{e x t}$. Therefore, the CG-RBC membrane model predicted lower limit of $\mu_{0, \text { Echinocyte }}$ is approximately $2.0 \mu_{0, \text { Discocyte }}\left(=8.0 \mu \mathrm{Nm}^{-1}\right)$ for optical tweezers stretching deformation scenario, and the echinocyte indicates stiffer nature than the discocyte when its membrane modulus is increased above $2.0 \mu_{0, \text { Discocyte }}$. This estimation of echinocyte membrane shear modulus agrees with experimentally extracted echinocyte membrane shear modulus [15, 69].

The spectrin link extensibility $(x)$ also affects the deformability of RBCs, and the limit of $x_{0, E c h i n o c y t e}$ such that echinocyte indicates reduced deformability is investigated using CG-RBC membrane model through optical tweezers stretching deformation. Several $x$ values such that $x=\left[1.0 x_{0, \text { Discocyte }}\right.$, $\left.1.5 x_{0, \text { Discocyte }}\right]$ are achieved at 0.05 multiplications of $x_{0, \text { Discocyte }}\left(x_{0, \text { Discocyte }}=0.45\right)$. All the other model parameters are equal to the corresponding model parameters as in section 3.1. The stretching deformation of echinocyte is investigated for $F^{\text {ext }}(\mathrm{pN})=[0,200]$ at above-mentioned $x$, and the evolution of $E I$ is compared against that of discocyte $E I$ at $x_{0, \text { Discoyte }}$. Figure 13 (b) represents the comparison of $E I$ of the echinocyte cell at $x=1.15 x_{0, \text { Discocyte }}, 1.20 x_{0, \text { Discocyte }}$ and $1.25 x_{0, \text { Discocyte }}$ against the $E I$ of discocyte at $x_{0, D i s c o c y t e}$. The echinocyte cell indicates lower deformability than that of discocyte at $x=1.20 x_{0, \text { Discocyte }}$ for high $F^{\text {ext }}$. In addition, the echinocyte deformability is lower than that of discocyte at $x=1.25 x_{0, \text { Discocyte }}$ for moderate to high $F^{\text {ext }}$. Echinocyte indicates more reduced deformability in a wider range of $F^{\text {ext }}$ at $x>1.25 x_{0, \text { Discocyte }}$. Therefore, the CG-RBC membrane model suggested a lower limit of $x_{0, E c h i n o c y t e}$ is $1.20 x_{0, \text { Discocyte }}(=0.54)$ such that its deformation behaviour agrees with the experimentally observed stiffer nature $[15,69,70]$. This study gives valuable insight to probable RBC membrane alterations, which may occur during echinocytosis such that the deformability of the RBC is decreased. However, the understanding on mechanisms behind these alterations requires extensive problem specific studies.
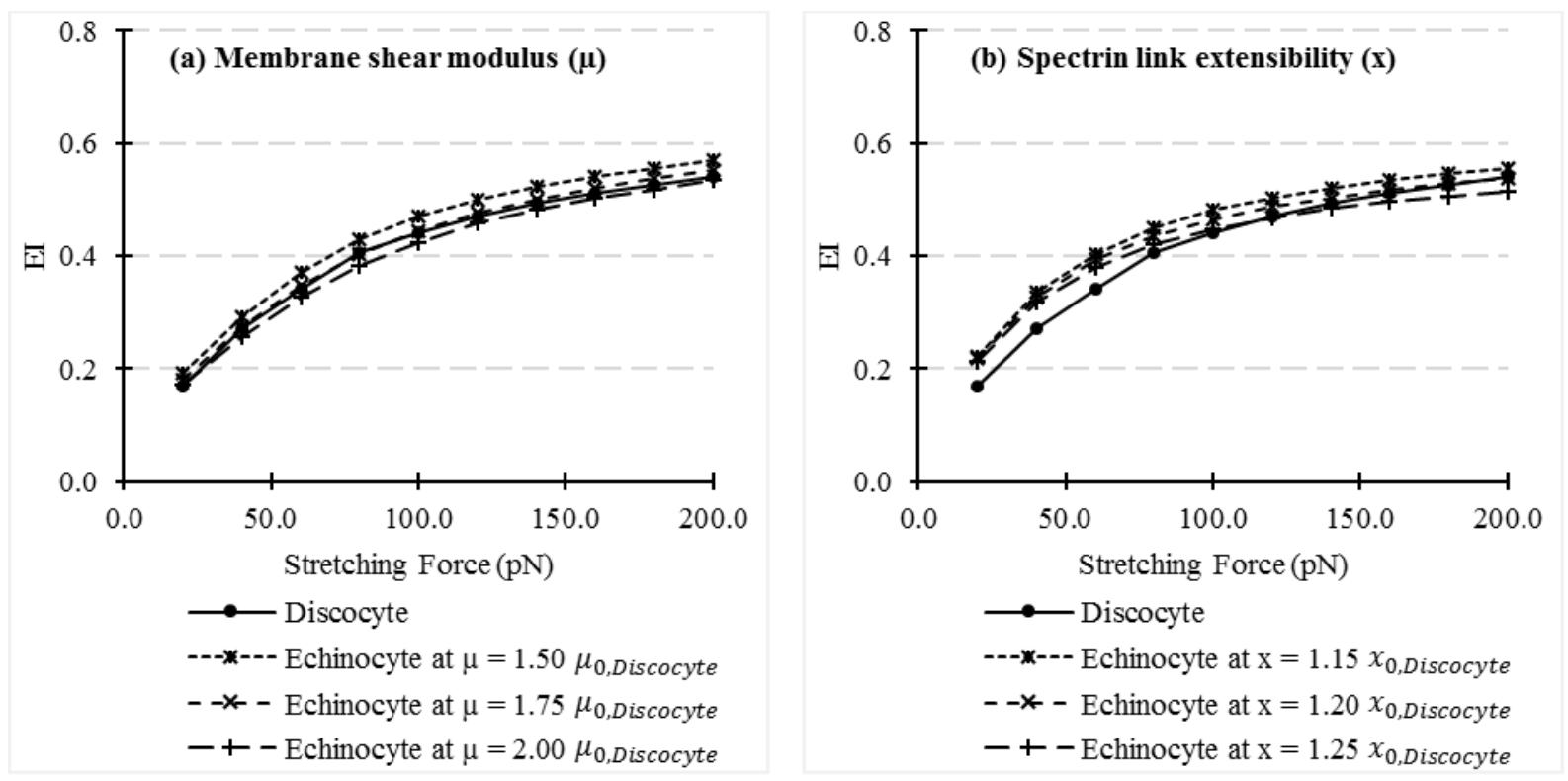
Figure 13 - Evolution of the elongation indices $(E I)$ of echinocyte cell undergoing optical tweezers stretching at varying (a) membrane shear modulus $(\mu)$, and (b) spectrin link extensibility $(x)$. The lower limits of $\mu_{0, E c h i n o c y t e}$ and $x_{0, E c h i n o c y t e}$ are estimated such the deformability of echinocyte decreases more than that of a discocyte.

In addition, the evolution of $E I$ is investigated at several $\kappa$ values such that $\kappa=\left[0.75 \kappa_{0}, 1.5 \kappa_{0}\right]$ where $\kappa_{0}$ is the bending modulus adopted in section 3.1. In general, $\kappa$ has limited influence (refer to Figure 12 (c)) on RBC deformability under optical tweezers stretching deformation conditions, and agrees with other numerical studies as well [2]. However, the increase in $\kappa$ results in a negligible decrement in cell deformability, and vice versa. This is due to the $E_{\text {Bending }}$ of the RBC membrane which influence the effort required to produce localized membrane curvature in the presence of optical tweezers stretching forces. Similarly, the influence of variations in the RBC membrane-bead contact area is investigated at several $d_{C}^{\prime}$ values such that $d_{C}^{\prime}(\mu \mathrm{m})=\left[0.75 d_{C}, 1.5 d_{C}\right]$ where $d_{C}$ is the RBC membrane-bead contact diameter adopted in section 3.1. Figure 12 (d) represents the evolution of $E I$ at $d_{C}^{\prime}=0.75 d_{C}, 1.0 d_{C}$ and $1.5 d_{C}$ for discocyte cell shape. In general, the change in $d_{C}^{\prime}$ inversely affects the RBC deformability, though negligible. Therefore, different RBC cell shapes indicate equivalent deformability irrespective of the number of cytoskeletal actin junctions on which the optical tweezers stretching forces. The characteristics of the $E I$ evolution do not change with changes in $\mu, \kappa$ and $d_{C}^{\prime}$, and only results in shifting of the curve. Therefore, these model parameters indicate a linear influence on the cell stretching behaviour irrespective of the cell morphology. This insight is valuable to understand the reliability of optical tweezers stretching experiments at varied RBC membrane-bead contact diameter, as $d_{C}^{\prime}$ has not always been mentioned [2, 23, 44].

The findings of the present study on parametric analyses of CG-RBC membrane model parameters and RBC membrane-bead contact diameter indicate that the cell deformability under optical tweezers stretching condition is primarily governed by the cytoskeletal spectrin network characteristics. Therefore, any modification to cytoskeleton would affect RBC deformability. The increased crosslinking of spectrin and actin [79], and increase in the average length of spectrin links [80] are cytoskeletal structural modification that lead to reduced cell deformability. However, problem specific investigations may provide detailed insight into probable structural modifications and/or mechanisms behind deformability changes of a RBC. 


\section{Summary and Outlook}

A numerical approach based on a CG-RBC membrane model is presented to investigate systematically the deformation behaviour of stomatocyte, discocyte and echinocyte RBC morphologies during optical tweezers stretching. It is hypothesized that different RBC morphologies and associated changes in cell deformability can be attributed to a BCM based membrane free energy model in order to investigate the cell deformation characteristics during optical tweezers stretching. The CG-RBC membrane model [61] captures the essential features of stomatocyte, discocyte and echinocyte morphologies, and enables the investigations to be performed at modest computational cost. It produces agreeable discocyte deformation behaviour with comparison to analogous experimental observations, and facilitates successful predictions on stomatocyte and echinocyte deformation characteristics. The predicted deformation behaviour confirms the lowered deformability of stomatocyte and echinocyte morphologies in comparison to healthy biconcave discocyte morphology. In addition, the results indicate the preservation of relevant morphological characteristics, the changes in spectrin link densities and the significant contribution of cytoskeletal spectrin network on deformation behaviour of RBC during stretching. Differences in cytoskeletal spectrin network remodelling is observed with respect to the cell morphology and applied stretching forces. The spectrin link extensibility inhibits the cell stretching at higher stretching forces and protects the cell from rupture.

RBC deformability is a critical characteristic to safeguard the cell at extensive and repeated deformation during its passage through narrow capillaries of the microvasculature. Therefore, improved understanding on the interrelation between RBC deformation characteristics and cell morphology under varying loading configurations, disease states, RBC storage lesion and cell shape-transformation scenarios is essential for better diagnostics and treatments. Optical tweezers method can be successfully used to study the changes in cell deformability under varying pathophysiological conditions, and the present numerical model provides a strengthened approach for such studies. The present numerical approach can be extended for a detailed discussion of the relationship between RBC morphology and its membrane mechanics, where any associated changes to the lipid-bilayer and/or cytoskeletal spectrin network should be considered, and is currently being employed to investigate the deformability characteristics and recoverability of RBCs undergoing storage lesion. 


\section{Author Contributions}

Geekiyanage developed the CG-RBC model and algorithm, performed modelling, data analysis and composed the manuscript. Sauret guided the model development, data analysis and revised the manuscript. Saha revised the manuscript. Flower revised the manuscript. Gu guided the model development and revised the manuscript.

\section{Conflicts of Interests}

There are no conflicts of interests to declare.

\section{Acknowledgements}

The authors would like to acknowledge the Australian Research Council for their financial support through Linkage Grant (LP150100737), and Queensland University of Technology (QUT) for the financial assistance through QUT Postgraduate Research Award (QUTPRA), Higher Degree Research (HDR) Tuition Fee Sponsorship and QUT Excellence Top-Up Scholarship. Support provided by Dr. HN Polwaththe-Gallage, Dr. S Barns, QUT's High Performance Computer Resources (HPC) and the Australian Red Cross Blood Service are gratefully acknowledged. The Australian Government fully fund the Australian Red Cross Blood Service for the provision of blood, blood products and services to the Australian community. 


\section{References}

1. Dao, M., J. Li, and S. Suresh, Molecularly based analysis of deformation of spectrin network and human erythrocyte. Materials Science and Engineering: C, 2006. 26(8): p. 1232-1244.

2. Dao, M., C.T. Lim, and S. Suresh, Mechanics of the human red blood cell deformed by optical tweezers. Journal of the Mechanics and Physics of Solids, 2003. 51(11): p. 2259-2280.

3. Kuzman, D., et al., Elastic properties of the red blood cell membrane that determine echinocyte deformability. European Biophysics Journal, 2004. 33(1): p. 1-15.

4. $\quad$ Kim, Y., K. Kim, and Y.K. Park, Measurement Techniques for Red Blood Cell Deformability: Recent Advances. 2012: INTECH Open Access Publisher.

5. Chang, H.-Y., et al., MD/DPD Multiscale Framework for Predicting Morphology and Stresses of Red Blood Cells in Health and Disease. PLOS Computational Biology, 2016. 12(10): p. e1005173.

6. Li, X., et al., Biomechanics and biorheology of red blood cells in sickle cell anemia. Journal of Biomechanics, 2016.

7. Li, H., et al., Mechanics of diseased red blood cells in human spleen and consequences for hereditary blood disorders. Proceedings of the National Academy of Sciences, 2018. 115(38): p. 9574.

8. Lim, C.T., et al., Large deformation of living cells using laser traps. Acta Materialia, 2004. 52(7): p. 1837-1845.

9. $\quad$ Mills, J.P., et al., Nonlinear elastic and viscoelastic deformation of the human red blood cell with optical tweezers. Mech Chem Biosyst, 2004. 1(3): p. 169-80.

10. Fedosov, D.A., et al., Computational Biorheology of Human Blood Flow in Health and Disease. Annals of Biomedical Engineering, 2014. 42(2): p. 368-387.

11. Fedosov, D.A., H. Noguchi, and G. Gompper, Multiscale modeling of blood flow: from single cells to blood rheology. Biomechanics and Modeling in Mechanobiology, 2014. 13(2): p. 239258.

12. Jiang, L.-G., et al., Coarse-grained molecular dynamics simulation of a red blood cell. Chinese Physics Letters, 2010. 27(2).

13. Polwaththe-Gallage, H.N., S.C. Saha, and Y. Gu, Deformation of a three-dimensional red blood cell in a stenosed microcapillary, in 8th Australasian Congress on Applied Mechanics (ACAM 8). 2014: Melbourne, Australia.

14. Yazdani, A., X. Li, and G.E. Karniadakis, Dynamic and rheological properties of soft biological cell suspensions. Rheologica Acta, 2016. 55(6): p. 433-449.

15. Park, Y., et al., Measurement of red blood cell mechanics during morphological changes. Proceedings of the National Academy of Sciences of the United States of America, 2010. 107(15): p. 6731-6736.

16. Israelachvili, J.N., Intermolecular and Surface Forces. 3 ed. 2011: Elsevier.

17. Iglič, A., V. Kralj-Iglič, and H. Hägerstrand, Amphiphile induced echinocyte-spheroechinoeyte transformation of red blood cell shape. European Biophysics Journal, 1998. 27(4): p. 335-339.

18. Mukhopadhyay, R., G. Lim, and M. Wortis, Echinocyte shapes: Bending, stretching, and shear determine spicule shape and spacing. Biophysical Journal, 2002. 82(4): p. 1756-1772.

19. Rudenko, S.V., Erythrocyte morphological states, phases, transitions and trajectories. Biochimica et Biophysica Acta - Biomembranes, 2010. 1798(9): p. 1767-1778.

20. Rudenko, S.V. and M.K. Saeid, Reconstruction of erythrocyte shape during modified morphological response. Biochemistry (Moscow), 2010. 75(8): p. 1025-1031. 
21. Etcheverry, S., et al., Real-time study of shape and thermal fluctuations in the echinocyte transformation of human erythrocytes using defocusing microscopy. Journal of Biomedical Optics, 2012. 17(10).

22. Chen, M. and F.J. Boyle, An Enhanced Spring-Particle Model for Red Blood Cell Structural Mechanics: Application to the Stomatocyte-Discocyte-Echinocyte Transformation. Journal of Biomechanical Engineering, 2017. 139(12): p. 121009-1-121009-11.

23. Li, Y., et al., Mechanical property analysis of stored red blood cell using optical tweezers. Colloids and Surfaces B: Biointerfaces, 2009. 70(2): p. 169-173.

24. Zheng, Y., et al., Characterization of red blood cell deformability change during blood storage. Lab on a Chip, 2014. 14(3): p. 577-583.

25. Czerwinska, J., et al., Red Blood Cell Aging During Storage, Studied Using Optical Tweezers Experiment. Cellular and Molecular Bioengineering, 2015. 8(2): p. 258-266.

26. $\mathrm{Xu}, \mathrm{Z}$., et al., Stiffness increase of red blood cells during storage. Microsystems \&Amp; Nanoengineering, 2018. 4: p. 17103.

27. Peng, Z., R.J. Asaro, and Q. Zhu, Multiscale simulation of erythrocyte membranes. Physical Review E, 2010. 81(3): p. 031904.

28. Wang, Y., et al., The mechanical properties of stored red blood cells measured by a convenient microfluidic approach combining with mathematic model. Biomicrofluidics, 2016. 10(2): p. 024104-024104.

29. Tomaiuolo, G., Biomechanical properties of red blood cells in health and disease towards microfluidics. Biomicrofluidics, 2014. 8(5): p. 051501.

30. Matthews, K., et al., Microfluidic deformability analysis of the red cell storage lesion. Journal of Biomechanics, 2015. 48(15): p. 4065-4072.

31. Muñoz, S., et al., Elastic energy of the discocyte-stomatocyte transformation. Biochimica et Biophysica Acta (BBA) - Biomembranes, 2014. 1838(3): p. 950-956.

32. Li, H. and G. Lykotrafitis, Erythrocyte Membrane Model with Explicit Description of the Lipid Bilayer and the Spectrin Network. Biophysical Journal, 2014. 107(3): p. 642-653.

33. Wong, P., A basis of echinocytosis and stomatocytosis in the disc-sphere transformations of the erythrocyte. Journal of Theoretical Biology, 1999. 196(3): p. 343-361.

34. Fedosov, D.A., B. Caswell, and G.E. Karniadakis, Systematic coarse-graining of spectrin-level red blood cell models. Computer Methods in Applied Mechanics and Engineering, 2010. 199(29-32): p. 1937-1948.

35. Fedosov, D.A., B. Caswell, and G.E. Karniadakis, Coarse-Grained Red Blood Cell Model with Accurate Mechanical Properties, Rheology and Dynamics, in Annual International Conference of the IEEE Engineering in Medicine and Biology Society. 2009. p. 4266-4269.

36. Fedosov, D.A., B. Caswell, and G.E. Karniadakis, A Multiscale Red Blood Cell Model with Accurate Mechanics, Rheology, and Dynamics. Biophysical Journal, 2010. 98(10): p. 22152225 .

37. Zhangli Peng, Robert J. Asaro, and Q. Zhu, Multiscale modelling of erythrocytes in Stokes flow. Journal of Fluid Mechanics, 2011.

38. Peng, Z., et al., Lipid bilayer and cytoskeletal interactions in a red blood cell. Proceedings of the National Academy of Sciences of the United States of America, 2013. 110(33): p. 1335613361.

39. Ju, M., et al., A review of numerical methods for red blood cell flow simulation. Computer Methods in Biomechanics and Biomedical Engineering, 2015. 18(2): p. 130-140. 
40. Monzel, C. and K. Sengupta, Measuring shape fluctuations in biological membranes. Journal of Physics D-Applied Physics, 2016. 49(24): p. 21.

41. Bento, D., et al., Deformation of Red Blood Cells, Air Bubbles, and Droplets in Microfluidic Devices: Flow Visualizations and Measurements. Micromachines, 2018. 9(4): p. 151.

42. Polwaththe-Gallage, H.N., et al., SPH-DEM approach to numerically simulate the deformation of three-dimensional RBCs in non-uniform capillaries. Biomed Eng Online, 2016. 15(Suppl 2): p. 161.

43. Barns, S., et al., Investigation of red blood cell mechanical properties using AFM indentation and coarse-grained particle method. Biomed Eng Online, 2017. 16(1): p. 140.

44. Hénon, S., et al., A new determination of the shear modulus of the human erythrocyte membrane using optical tweezers. Biophysical journal, 1999. 76(2): p. 1145-1151.

45. Liang, Y., et al. Cell deformation and assessment with tunable "tug-of-war" optical tweezers. in Conference on Lasers and Electro-Optics. 2019. San Jose, California: Optical Society of America.

46. Sheetz, M.P. and S.J. Singer, Biological membranes as bilayer couples: Molecular mechanisms of drug-erythrocyte interactions. Proceedings of the National Academy of Sciences of the United States of America, 1974. 71(11): p. 4457-4461.

47. Tachev, K.D., K.D. Danov, and P.A. Kralchevsky, On the mechanism of stomatocyteechinocyte transformations of red blood cells: Experiment and theoretical model. Colloids and Surfaces B: Biointerfaces, 2004. 34(2): p. 123-140.

48. Gedde, M.M., E. Yang, and W.H. Huestis, Resolution of the paradox of red cell shape changes in low and high $\mathrm{pH}$. Biochimica et Biophysica Acta (BBA) - Biomembranes, 1999. 1417(2): p. 246-253.

49. Miao, L., et al., Budding transitions of fluid-bilayer vesicles: The effect of area-difference elasticity. Physical Review E, 1994. 49(6): p. 5389-5407.

50. Udo Seifert, Karin Berndl, and R. Lipowsky, Shape transformations of vesicles: Phase diagram for spontaneous- curvature and bilayer-coupling models. Physical Review A, 1991. 44(2): p. 1182-1202.

51. Brecher, G. and M. Bessis, Present Status of Spiculed Red Cells and Their Relationship to the Discocyte-Echinocyte Transformation: A Critical Review. Blood, 1972. 40(3): p. 333.

52. Bessis, M. Red Cell Shapes. An Illustrated Classification and its Rationale. in Red Cell Shape. 1973. Berlin, Heidelberg: Springer Berlin Heidelberg.

53. Svetina, S. and B. Žekš, Membrane bending energy and shape determination of phospholipid vesicles and red blood cells. European Biophysics Journal, 1989. 17(2): p. 101-111.

54. Lim H. W, G., M. Wortis, and R. Mukhopadhyay, Stomatocyte-discocyte-echinocyte sequence of the human red blood cell: Evidence for the bilayer-couple hypothesis from membrane mechanics. Proceedings of the National Academy of Sciences of the United States of America, 2002. 99(26): p. 16766-16769.

55. Svetina, S., et al., The cooperative role of membrane skeleton and bilayer in the mechanical behaviour of red blood cells. Bioelectrochemistry, 2004. 62(2): p. 107-113.

56. Khairy, K., J. Foo, and J. Howard, Shapes of Red Blood Cells: Comparison of 3D Confocal Images with the Bilayer-Couple Model. Cellular and Molecular Bioengineering, 2008. 1(2): p. 173.

57. Pages, G., T.W. Yau, and P.W. Kuchel, Erythrocyte shape reversion from echinocytes to discocytes: Kinetics via fast-measurement NMR diffusion-diffraction. Magnetic Resonance in Medicine, 2010. 64(3): p. 645-652. 
58. Khairy, K. and J. Howard, Minimum-energy vesicle and cell shapes calculated using spherical harmonics parameterization. Soft Matter, 2011. 7(5): p. 2138-2143.

59. Tong, Z.-X., et al., Coarse-grained area-difference-elasticity membrane model coupled with $I B-L B$ method for simulation of red blood cell morphology. Physica A: Statistical Mechanics and its Applications, 2018. 509: p. 1183-1194.

60. Lim H. W, G., M. Wortis, and R. Mukhopadhyay, Red Blood Cell Shapes and Shape Transformations: Newtonian Mechanics of a Composite Membrane: Sections 2.5-2.8, in Soft Matter. 2009, Wiley-VCH Verlag GmbH \& Co. KGaA. p. 83-250.

61. Geekiyanage, N.M., et al., A coarse-grained red blood cell membrane model to study stomatocyte-discocyte-echinocyte morphologies. PLOS ONE, 2019. 14(4): p. e0215447.

62. Helfrich, W., Blocked lipid exchange in bilayers and its possible influence on the shape of vesicles. Z Naturforsch C, 1974. 29c(9-10): p. 510-5.

63. Li, X., et al., Computational Biomechanics of Human Red Blood Cells in Hematological Disorders. Journal of Biomechanical Engineering, 2017. 139(2): p. 021008-1-021008-13.

64. Nakamura, M., S. Bessho, and S. Wada, Analysis of red blood cell deformation under fast shear flow for better estimation of hemolysis. International Journal for Numerical Methods in Biomedical Engineering, 2014. 30(1): p. 42-54.

65. Mohandas, N. and P.G. Gallagher, Red cell membrane: past, present, and future. Blood, 2008. 112(10): p. 3939-3948.

66. Suresh, S., et al., Connections between single-cell biomechanics and human disease states: gastrointestinal cancer and malaria. Acta Biomaterialia, 2005. 1(1): p. 15-30.

67. Svetina, S., Vesicle budding and the origin of cellular life. Chemphyschem, 2009. 10(16): p. 2769-76.

68. Heinrich, V., S. Svetina, and B. Žekš, Nonaxisymmetric vesicle shapes in a generalized bilayercouple model and the transition between oblate and prolate axisymmetric shapes. Physical Review E, 1993. 48(4): p. 3112-3123.

69. Kozlova, E., et al., Morphology, membrane nanostructure and stiffness for quality assessment of packed red blood cells. Scientific Reports, 2017. 7(1): p. 7846.

70. Roussel, C., et al., Spherocytic shift of red blood cells during storage provides a quantitative whole cell-based marker of the storage lesion. Transfusion, 2017.

71. $\mathrm{Xu}, \mathrm{Z}$., et al. Microfluidic measurement of RBC bending stiffness changes in blood storage. in 2017 19th International Conference on Solid-State Sensors, Actuators and Microsystems (TRANSDUCERS). 2017.

72. Li, J., et al., Spectrin-Level Modeling of the Cytoskeleton and Optical Tweezers Stretching of the Erythrocyte. Biophysical Journal, 2005. 88(5): p. 3707-3719.

73. Chen, X.Y., et al., Membrane surface charge and morphological and mechanical properties of young and old erythrocytes. Current Applied Physics, 2007. 7(SUPPL.1): p. e94-e96.

74. Li, J., et al., Cytoskeletal dynamics of human erythrocyte. Proceedings of the National Academy of Sciences of the United States of America, 2007. 104(12): p. 4937-4942.

75. Závodszky, G., et al., Cellular Level In-silico Modeling of Blood Rheology with An Improved Material Model for Red Blood Cells. Frontiers in Physiology, 2017. 8: p. 563.

76. Boey, S.K., D.H. Boal, and D.E. Discher, Simulations of the Erythrocyte Cytoskeleton at Large Deformation. I. Microscopic Models. Biophysical Journal, 1998. 75(3): p. 1573-1583.

77. Discher, D.E., D.H. Boal, and S.K. Boey, Simulations of the Erythrocyte Cytoskeleton at Large Deformation. II. Micropipette Aspiration. Biophysical Journal, 1998. 75(3): p. 1584-1597. 
78. Lee, J.C.M. and D.E. Discher, Deformation-Enhanced Fluctuations in the Red Cell Skeleton with Theoretical Relations to Elasticity, Connectivity, and Spectrin Unfolding. Biophysical Journal, 2001. 81(6): p. 3178-3192.

79. Branton, D., C.M. Cohen, and J. Tyler, Interaction of cytoskeletal proteins on the human erythrocyte membrane. Cell, 1981. 24(1): p. 24-32.

80. Lai, L., et al., Stiffening of Red Blood Cells Induced by Cytoskeleton Disorders: A Joint TheoryExperiment Study. Biophysical Journal, 2015. 109(11): p. 2287-2294. 